\title{
2'-Behenoyl-Paclitaxel Conjugate Containing Lipid Nanoparticles for the Treatment of Metastatic Breast Cancer
}

\author{
Ping Ma ${ }^{1}$, S. Rahima Benhabbour ${ }^{1}$, Lan Feng ${ }^{1}$, and Russell J Mumper ${ }^{1,2, *}$ \\ ${ }^{1}$ Center for Nanotechnology in Drug Delivery, Division of Molecular Pharmaceutics, UNC \\ Eshelman School of Pharmacy, University of North Carolina at Chapel Hill, Chapel Hill, NC \\ 27599, USA \\ ${ }^{2}$ UNC Lineberger Comprehensive Cancer Center, University of North Carolina at Chapel Hill, NC, \\ USA
}

\begin{abstract}
The aim of these studies was to develop a novel 2'-behenoyl-paclitaxel (C22-PX) conjugate nanoparticle (NP) formulation for the treatment of metastatic breast cancer. A lipophilic paclitaxel derivative C22-PX was synthesized and incorporated into lipid-based NPs. Free C22-PX and its NP formulation were evaluated in a series of in-vitro and in-vivo studies. The results demonstrated that C22-PX NPs were much better tolerated and had significantly higher plasma and tumor AUCs compared to Taxol at the maximum tolerated dose (MTD) in a subcutaneous 4T1 mouse mammary carcinoma model. These benefits resulted in significantly improved antitumor efficacy with the NP-based formulation.
\end{abstract}

\section{Keywords}

paclitaxel; 2'-behenoyl-paclitaxel; nanoparticle; 4T1 mouse model; metastatic breast cancer

\section{Introduction}

Paclitaxel (PX) is one of the most effective chemotherapeutic anticancer drugs and is mainly used to treat lung, ovarian, and breast cancer [1]. Due to its low water solubility, it is formulated in a mixture of 50:50\% (v/v) polyoxyethylated castor oil (Cremophor EL) and dehydrated ethanol under the trademark "Taxol". However, Cremophor EL is known to be associated with serious side effects, such as hypersensitivity [2]. In addition, PX is known as a substrate of P-glycoprotein (P-gp), which could actively pump PX out of the cells and induce resistance [3]. To overcome these problems, several strategies have been applied, mainly including modified "Cremophor-free" formulations [4-7] and/or structure modification of the drug (e.g. PX analogs and prodrugs) [8-10].

\footnotetext{
(C) 2012 Elsevier Ireland Ltd. All rights reserved.

*Corresponding author: Russell J. Mumper, Ph.D., Vice Dean and John A. McNeill Distinguished Professor, Center for Nanotechnology in Drug Delivery, Division of Molecular Pharmaceutics, UNC Eshelman School of Pharmacy, CB\# 7355, 100G Beard Hall, University of North Carolina at Chapel Hill, Chapel Hill, North Carolina 27599-7355, mumper@email.unc.edu, Phone: (919) 966-1271, Fax: (919) 966-6919.

Publisher's Disclaimer: This is a PDF file of an unedited manuscript that has been accepted for publication. As a service to our customers we are providing this early version of the manuscript. The manuscript will undergo copyediting, typesetting, and review of the resulting proof before it is published in its final citable form. Please note that during the production process errors may be discovered which could affect the content, and all legal disclaimers that apply to the journal pertain.

Conflict of Interest Statement

None
} 
In recent years a great deal of research has been reported to formulate PX into different nanosystems, such as o/w emulsions [4], micelles [5], liposomes [6], and solid lipid nanoparticles [7]. These formulations have shown some benefits compared to Taxol both invitro and in-vivo, however, in most cases the solubilization capacity of PX in these formulations was limited and PX was released rapidly from the formulation. Furthermore, most of the formulations lack mechanisms to overcome P-gp-mediated drug resistance. Abraxane, a PX albumin-bound nanoparticle (NP) formulation with the particle size of $\sim 130$ $\mathrm{nm}$, was approved by the FDA in 2005 for the treatment of metastatic breast cancer. Due to the elimination of Cremophor EL and ethanol, this formulation demonstrates some advantages in terms of reduced toxicity compared to Taxol. In a phase III clinical trial, Abraxane demonstrated the improved response rates compared to Taxol in the treatment of metastatic breast cancer as a first-line therapy [11]. However, in this phase III study, Abraxane failed to show a statistically significant enhanced median survival compared to Taxol $(\mathrm{p}=0.374)$; therefore, whether Abraxane could improve survival is still unclear.

To potentially better target tumors and to address overcome P-gp-mediated drug resistance, our laboratory has developed a new technology to engineer stable oil-filled lipid-based PX BTM (Brij 78 and TPGS as the surfactants, and Miglyol 812 as the oil phase) NPs from oilin-water microemulsion precursors [12]. The loading capacity of the drug was up to $8 \%$ (drug/oil, w/w), and only $50 \%$ of the drug was released in PBS after $72 \mathrm{~h}$. This PX BTM NP system has shown significantly greater anticancer efficacy in nude mice bearing resistant NCI/ADR-RES tumors compared to Taxol. The mechanisms of PX BTM NPs to overcome $\mathrm{P}$-gp-mediated drug resistance were also investigated and shown to be due to P-gp inhibition and ATP depletion [13].

To further improve the lipophilicity of PX and facilitate its retention in the lipid core, modification of PX to a fatty acid-PX derivative is a potential approach. To date, many PX derivatives have been reported to increase its water solubility [10, 14-16], or oppositely, to enhance its lipophicility in order to entrap more drug into lipid-based formulations [17-20]. The most advanced fatty acid-PX derivative is docosahexaenoic acid-PX (DHA-PX) which is currently in phase III clinical trials [19, 21-23]. DHA is linked to PX through an ester bond on its C-2' position. The DHA-PX conjugate does not have microtubule assembly activity and is presumably non-toxic. The conjugate converts to active PX when metabolized by esterases in the body. The conjugate was shown to prolong exposure of PX and reduce its peak blood concentration, which allowed for 4.4-fold higher maximum tolerated dose (MTD) of DHA-PX as compared to Taxol in mice. In an M109 subcutaneous lung tumor mouse model, DHA-PX completely eliminated measurable tumors while PX did not result in even partial tumor regression at its MTD [19]. However, there are some issues associated with this conjugate: 1) it is still formulated in Cremophor EL/Ethanol; and 2) it remains a Pgp substrate [24]. In the current studies, a 2'-behenoyl-paclitaxel (C22-PX) conjugate was synthesized and formulated into our modified BTM NPs. A series of in-vitro studies were performed to compare C22-PX conjugate to PX. In-vivo MTD, antitumor efficacy, and pharmacokinetic and biodistribution studies of C22-PX NPs and Taxol were also conducted in a 4T1 subcutaneous mouse model.

\section{Materials and methods}

\subsection{Materials}

PX powder was purchased from LC Laboratories (Woburn, MA, USA). PX injection was bought from Hospira Inc. (Lake Forest, IL, USA). Polyoxyl 20-stearyl ether (Brij 78) was obtained from Uniqema (Wilmington, DE, USA). D-alpha tocopheryl polyethylene glycol 1000 succinate (TPGS) was generously provided by Eastman Chemical Co. (Kingsport, TN, USA). Behenoyl chloride, anhydrous dichloromethane (DCM), and 4- 
dimethylaminopyridine (DMAP) were purchased from Sigma (St. Louis, MO, USA). The tubulin polymerization assay kit was purchased from Cytoskeleton Inc. (Denver, CO, USA). 4T1 mouse mammary carcinoma cell line was obtained from ATCC (Manassas, VA, USA). The cell line was cultured in RPMI-1640 medium (Invitrogen, Carlsbad, CA, USA) containing 10\% fetal bovine serum (FBS) (ATCC, Manassas, VA, USA) and antibiotics at $37^{\circ} \mathrm{C}$ in $5 \% \mathrm{CO}_{2}$ humidified atmosphere.

\subsection{Synthesis and characterization of C22-PX}

A flame-dried round-bottom flask was charged with PX $\left(25 \mathrm{mg}, 2.9 \times 10^{-5} \mathrm{~mol}, 1\right.$ equiv) and DMAP (7.2 mg, $5.8 \times 10^{-5} \mathrm{~mol}, 2$ equiv) in anhydrous DCM (3 ml) under argon. The solution was stirred for $10 \mathrm{~min}$ at $0^{\circ} \mathrm{C}$. Behenoyl chloride $\left(11 \mathrm{mg}, 3.1 \times 10^{-5} \mathrm{~mol}, 1.05\right.$ equiv) was added, and the reaction mixture was stirred for $6 \mathrm{~h}$ at $0^{\circ} \mathrm{C}$ under argon. The reaction was monitored by TLC (EtOAc:hexanes $3: 1 \mathrm{v} / \mathrm{v}, \mathrm{R}_{\mathrm{f}}=0.74$ ) for completion. After completion, the solvent was removed by rotary evaporation in-vacuo and the crude product was dissolved in diethylether $(10 \mathrm{ml})$ and washed with $5 \% \mathrm{HCl}(3 \times 10 \mathrm{ml})$, and finally with brine $(10 \mathrm{ml})$ to remove the salt byproducts. The organic phase was dried over anhydrous sodium sulfate, and the solvent was evaporated in-vacuo. The final C22-PX conjugate (29.3 $\mathrm{mg}, 85 \%$ ) was obtained by silica-packed column chromatography purification with the solvent system of EtOAc:hexanes (3:1, v/v). ${ }^{1} \mathrm{H}$ NMR $\left(400 \mathrm{MHz}, \mathrm{CDCl}_{3}\right): \delta(\mathrm{ppm})=0.81$ (t, 3H, $\left.-\mathrm{CH}_{3}\left(\mathrm{CH}_{2}\right)_{20}\right), 1.06$ (s, 6H, $\left.-H_{16,17}\right), 1.18\left(\mathrm{~m}, 18 \mathrm{H},-\left(\mathrm{CH}_{2}\right)_{7} \mathrm{CH}_{2} \mathrm{CH}_{3}\right), 1.61(\mathrm{~s}, 3 \mathrm{H}$, $\left.-H_{19}\right), 1.72\left(\mathrm{~m}, 2 \mathrm{H},-H_{6}\right), 1.80\left(\mathrm{~m}, 2 \mathrm{H},-H_{14}\right), 1.87$ (s, 3H, $\left.-H_{18}\right), 2.15$ (s, 6H, $\left.-H_{22,31}\right)$, $2.38\left(\mathrm{~d}, 1 \mathrm{H},-H_{3}\right), 3.41\left(\mathrm{q}, 2 \mathrm{H},-H_{5,7}\right), 3.74\left(\mathrm{~s}, 1 \mathrm{H},-\mathrm{O} H_{7}\right), 3.76\left(\mathrm{~s}, 1 \mathrm{H},-\mathrm{O} H_{1}\right), 4.14(\mathrm{~d}, 1 \mathrm{H}$, $\left.-H_{20}\right), 4.23\left(\mathrm{~d}, 1 \mathrm{H},-H_{20}\right), 4,39\left(\mathrm{t}, 1 \mathrm{H},-H_{13}\right), 4.86\left(\mathrm{~d}, 1 \mathrm{H},-H_{2}\right), 5.22\left(\mathrm{~s}, 1 \mathrm{H},-H_{10}\right), 5.84(\mathrm{~d}$, $1 \mathrm{H},-H_{2}$ ), $6.81\left(\mathrm{~d}, 1 \mathrm{H},-H_{3}\right.$ ) $, 7.18-7.32\left(\mathrm{~m}, 5 \mathrm{H},-\mathrm{Ar}-\mathrm{H}_{33-37}\right), 7.32-35\left(\mathrm{~m}, 3 \mathrm{H}, \mathrm{Ar}-\mathrm{H}_{26-28}\right.$ ), 7.44 (m, 3H, Ar- $\left.H_{8}{ }^{\prime}-10^{\prime}\right), 7.66$ (d, $\left.2 \mathrm{H},-\mathrm{Ar}-H_{7}, 11^{\prime}\right), 8.05$ (d, $\left.2 \mathrm{H},-\mathrm{Ar}-H_{25,29}\right) .{ }^{13} \mathrm{C} \mathrm{NMR}$ $\left(100 \mathrm{MHz}, \mathrm{CD}_{3} \mathrm{OD}\right): \delta(\mathrm{ppm})=8.58\left(-C_{19}\right), 13.1\left(-\mathrm{CH}_{3}\left(\mathrm{CH}_{2}\right)_{20}\right), 13.8\left(-\mathrm{C}_{18}\right), 19.8\left(-C_{31}\right)$, $21.1\left(-C_{22}\right), 21.7\left(-\left(\mathrm{CH}_{2}\right)_{19} \mathrm{CH}_{2} \mathrm{CH}_{3}\right), 23.7\left(-C_{16,17}\right), 25.8\left(-\left(\mathrm{CH}_{2}\right)_{18} \mathrm{CH}_{2} \mathrm{CH}_{2} \mathrm{C}_{1}\right.$ "), 30.9 ($\mathrm{CH}_{3} \mathrm{CH}_{2} \mathrm{CH}_{2}\left(\mathrm{CH}_{2}\right)_{18} \mathrm{C}_{1}$ "), $32.8\left(-C_{6}\right), 34.5\left(-C_{14}\right), 42.1\left(-C_{15}\right), 44.6\left(-C_{3}\right), 51.8\left(-C_{3},\right)$, $57.5\left(-C_{8}\right), 70.7\left(-C_{13}\right), 71.1\left(-C_{7}\right), 72.8\left(-C_{2}\right), 74.1\left(-C_{10}\right), 74.6\left(-C_{20}\right), 76.3\left(-C_{6}\right), 78.2$ $\left(-C_{1}\right), 80.1\left(-C_{4}\right), 83.4\left(-C_{5}\right), 125.5\left(-C_{33,35,37}\right), 126.1\left(-C_{7}, 11^{\prime}\right), 127.4-128.2\left(-C_{26,28},-\right.$ $\left.C_{34,36},-C_{8}, 10^{\prime}\right), 129.2\left(-C_{24,25,29}\right), 132.6\left(-C_{27}\right), 131.0\left(-C_{9}\right), 131.8\left(-C_{27}\right), 132.6\left(-C_{32}\right)$, $\left.136.0\left(-C_{11}\right), 141.8\left(-C_{12}\right), 166.1\left(-C_{23}\right), 167.1\left(-C_{5}\right), 168.8\left(-C_{21},-C_{30}\right), 170.2\left(-C_{1}\right)^{\prime}\right)$, $171.5\left(-C_{1}\right.$ "), $202.8\left(-C_{9}\right)$. Mass spectrometry analysis also provided evidence for successful preparation of the C22-PX conjugate. The mass of the observed molecular ion clearly corresponded to the $\mathrm{Na}^{+}$adduct of the conjugate, with no observable lower molecular weight fragments or starting material. Calc.: $[\mathrm{M}]^{+} \mathrm{m} / \mathrm{z}=1176.47$. Found LC-MS: $\left[\mathrm{M}+\mathrm{Na}^{+}\right]$ $=1198.13$.

\subsection{Quantitative analysis of PX and C22-PX}

The concentration of PX was determined by HPLC/MS/MS system. The HPLC system comprised a Finnigan Surveyor Autosampler Plus and a Finnigan Surveyor MS Pump Plus (Thermo Scientific). Chromatographic separations were achieved using a SunFire ${ }^{\mathrm{TM}} \mathrm{C} 18$ column $(2.1 \times 30 \mathrm{~mm}, 3.5 \mu \mathrm{m}$ particle size, Waters $)$ at $25^{\circ} \mathrm{C}$. The mobile phase consisted of the mixtures of methanol (solvent A) and $0.1 \%$ formic acid in deionized water (solvent B). The elution protocol consisted of a 55:45 ratio $(\mathrm{v} / \mathrm{v})$ of solvent A to solvent $\mathrm{B}$ for $0.5 \mathrm{~min}$, followed by a gradient starting at 70:30 (A to B, v/v) over $6 \mathrm{~min}$ and finishing at 55:45 (A to $\mathrm{B}, \mathrm{v} / \mathrm{v}$ ) over $8 \mathrm{~min}$. The flow rate was $0.5 \mathrm{ml} / \mathrm{min}$. Mass spectrometric analysis was performed by Thermo Scientific TSQ Quantum Access with positive ionization. The capillary temperature was set to $390^{\circ} \mathrm{C}$, and the spray voltage was $4000 \mathrm{~V}$. M/z $876.3 \rightarrow$ 308.0 for PX and $\mathrm{m} / \mathrm{z} 830.0 \rightarrow 549.0$ for docetaxel were used for quantification of analytes. Docetaxel was added as the internal standard. To quantify PX concentration in plasma samples, solid phase extraction was applied before the LC/MS assay. A Waters Oasis ${ }^{\circledR}$ HLB 
cartridge $(1 \mathrm{cc} / 10 \mathrm{mg})$ was used and conditioned by $20 \%$ methanol in dichloromethane, methanol and water, then the plasma samples were loaded on the cartridge and washed by $5 \%$ methanol and 55\% methanol in water, respectively. The collection samples were then eluted by $20 \%$ methanol in dichloromethane, after which the organic solvent was removed by rotary evaporation and the resulting solid was dissolved in the mobile phase for LC/MS analysis.

The concentration of C22-PX was determined by HPLC analysis using an Inertsil ODS-3 column $(4.6 \times 150 \mathrm{~mm}, 3 \mu \mathrm{m}$ particle size, GL Sciences) at the wavelength of $230 \mathrm{~nm}$. The mobile phase was acetonitrile/water/isopropanol (35:5:60, v/v/v) with a flow rate of $1.0 \mathrm{ml} /$ $\mathrm{min}$. For the plasma or tissue samples, C22-PX was extracted from the samples by single extraction with methanol/acetonitrile $(2: 1, \mathrm{v} / \mathrm{v})$. The mixtures were then centrifuged at $14,000 \mathrm{~g}$ for $5 \mathrm{~min}$, and the supernatant was collected and analyzed by HPLC assay.

\subsection{Solubility of PX and C22-PX in different Miglyols}

Excess PX or C22-PX powder was added into Miglyol 808, 810, 812, or 818, respectively, and stirred at room temperature for $24 \mathrm{~h}$. The samples were subsequently centrifuged at $25^{\circ} \mathrm{C}$ at the speed of $14,000 \mathrm{~g}$ for $20 \mathrm{~min}$. The supernatant was accurately weighed and dissolved in methanol in a $50 \mathrm{ml}$ volumetric flask. The concentration of PX or C22-PX in the flask was determined by LC-MS or HPLC analysis, respectively.

\subsection{Tubulin polymerization activity of PX and C22-PX}

A tubulin polymerization assay kit was purchased from Cytoskeleton Inc. (Cat\# BK006P). The assay is based on the principle that the light scattered by microtubules is proportional to the concentration of tubulin polymer $[25,26]$. The standard (control) polymerization reaction contains $100 \mu \mathrm{l}$ of $3 \mathrm{mg} / \mathrm{ml}$ tubulin in $80 \mathrm{mM}$ PIPES pH 6.9, $0.5 \mathrm{mM}$ EGTA, $2 \mathrm{mM}$ $\mathrm{MgCl}_{2}, 1 \mathrm{mM}$ GTP, and $10 \%$ glycerol. The assay was conducted at $37^{\circ} \mathrm{C}$ and the tubulin polymerization reaction was monitored by the change in absorbance at $340 \mathrm{~nm}$ with a temperature controlled Synergy 2 Multi-Detection Microplate Reader (BioTek, Winooski, VT, USA). C22-PX concentrations of 5, 10, and $20 \mu \mathrm{M}$ were applied in the assay. PX (10 $\mu \mathrm{M}$ ) was used as the positive control.

\subsection{In-vitro cytotoxicity of PX and C22-PX}

The cytotoxicity of C22-PX and PX in 4T1 mouse mammary carcinoma cells was determined using a MTT (3-[4,5-dimethylthiazol-2-yl]-2,5-diphenyl tetrazolium bromide) assay [27]. Briefly, the viability of 4T1 cells was determined by trypan blue staining (>95\% cell viability for experiments) and the cells were counted and seeded in 96-well plates at 5,000 cells $/ 100 \mu \mathrm{l}$ growth medium. The C22-PX concentrations of 100, 250, 500, 1,000, $5,000,10,000$, and $25,000 \mathrm{nM}$, and PX concentrations of 1, 10,50, 100, 250, 1,000, and $5,000 \mathrm{nM}$, respectively, were applied and added to the plate $(100 \mu \mathrm{l} / \mathrm{well})$ at $37^{\circ} \mathrm{C}$ in $5 \%$ $\mathrm{CO}_{2}$ for $48 \mathrm{~h}$. The cells were then incubated with MTT stock solution $(5 \mathrm{mg} / \mathrm{ml}$ in PBS; pH 7.4) at $37^{\circ} \mathrm{C}$ for another $4 \mathrm{~h}$. Next, the medium was removed and the converted dye was solubilized with the addition of DMSO. The absorbance was measured using a Synergy 2 Multi-Detection Microplate Reader at $570 \mathrm{~nm}$, and the concentration of drug that inhibited cell survival by $50 \%$ ( $\mathrm{IC}_{50}$ ) was determined from cell survival plots.

\subsection{Optimization and characterization of C22-PX NPs}

Our laboratory has developed "nanotemplate engineering" technology to successfully prepare PX BTM NPs with a particle size of $200 \mathrm{~nm}$ [12]. Since C22-PX is more lipophilic and to some extent exhibits a surfactant property, the current C22-PX BTM NP formulations were optimized by decreasing the amount of surfactants of Brij 78 and TPGS used in the 
previous BTM formulation. In brief, Miglyol 812, Brij 78 and TPGS were first weighed in a glass vial, and the vial was then heated to $65^{\circ} \mathrm{C}$ to melt the oil and surfactants while stirring. Next, C22-PX, dissolved in ethanol, was transferred to the vial and ethanol was removed by nitrogen gas. Finally, preheated deionized water was added into the vial to form a NP suspension via the microemulsion precursor. C22-PX micelles were prepared in the same manner as the NP formulation but without the oil phase of Miglyol 812. The particle size of the NPs was determined by photon correlation spectroscopy (PCS) using a Coulter N5 Plus Sub-Micron Particle Sizer (Beckman Coulter, Miami, FL, USA) at a fixed angle of $90^{\circ}$ and a temperature of $25^{\circ} \mathrm{C}$. The optimal NP formulation was defined as the one with a narrow particle size distribution of less than $200 \mathrm{~nm}$, high drug entrapment efficiency in NPs, and physical stability upon storage at $4^{\circ} \mathrm{C}$ or room temperature. The $\%$ drug entrapped in the NPs was determined by gel permeation chromatography (GPC) method. Briefly, the prepared NPs were applied to a $15 \mathrm{~cm}$ Sepharose CL-4B packed GPC column (Sigma, St. Louis, MO, USA) and eluted with PBS as the mobile phase. The NP fractions of 5-8 (1 ml per fraction) were collected and the total amount of C22-PX in these fractions was determined. The \% entrapment was calculated as follows: \% Entrapment $=[($ Mass of drug in NPs in GPC factions 5-8) / (Total mass of drug used to prepare formulation)] $\times 100 \%(w / w)$

\subsection{Stability of various C22-PX formulations in PBS at $37^{\circ} \mathrm{C}$}

Different C22-PX formulations, including free C22-PX, C22-PX NPs, and C22-PX micelles were incubated in PBS at $37^{\circ} \mathrm{C}$ in a water bath. At the scheduled time points of $1,4,10,24$, $48 \mathrm{~h}$, respectively, samples were collected and the concentration of C22-PX was determined by HPLC. Free PX was used as the control and the final concentrations of the drug in the experiment were $10 \mu \mathrm{g} / \mathrm{ml}$.

\subsection{C22-PX conversion to PX in tumor homogenates}

All animal procedures followed NIH guidelines and were performed under protocols approved by The University of North Carolina Institutional Animal Care and Use Committee. To test C22-PX conversion to the more active drug of PX, C22-PX and C22-PX NPs were spiked into fresh 4T1 tumor homogenate at the concentration of $10 \mu \mathrm{g} / \mathrm{g}$ tumor and incubated at $37^{\circ} \mathrm{C}$ in a water bath. To obtain $4 \mathrm{~T} 1$ tumor homogenate, a total of ten $\mathrm{BALB} / \mathrm{c}$ female mice from Charles River Laboratories (Wilmington, MA, USA) were first subcutaneously injected with $1 \times 10^{6} 4 \mathrm{~T} 1$ cells. When tumors reached a volume of 200-300 $\mathrm{mm}^{3}$, the mice were sacrificed and the tumors were removed. Next, the tumors were homogenized by Omni Bead Ruptor 24 Homogenizer (Kennesaw, GA, USA). At predetermined time points, $\sim 40-50 \mathrm{mg}$ of tumor homogenate was collected. The concentrations of both C22-PX and PX converted from C22-PX or C22-PX NPs were then determined by HPLC and LC-MS analysis, respectively.

\subsection{Maximum tolerated dose (MTD) studies of C22-PX NPs}

The MTD studies of Taxol, C22-PX NPs, and C22-PX micelles were conducted in 4T1 tumor-bearing mice. BALB/c female mice from Charles River Laboratories (Wilmington, MA, USA) were subcutaneously injected with $1 \times 10^{6} 4 \mathrm{~T} 1$ cells in the right flank. When the tumors reached sizes in the range of $50-150 \mathrm{~mm}^{3}$ ( 5 days after cell inoculation), the mice were injected by tail vein with different formulations with the treatment schedule of Q4d $\times$ 3. A total of 7 groups were used in the study (2-3 mice/group): 30 and $40 \mathrm{mg} P X / \mathrm{kg}$ of Taxol, 135 and $180 \mathrm{mg}$ PX/kg of C22-PX NPs, 90 and $120 \mathrm{mg}$ PX/mg of C22-PX micelles, and an untreated group as the control. Mice body weight change was closely monitored on a daily basis for two weeks. The MTD was defined as the allowance of body weight loss less than $15 \%$ at the highest dose, and neither mice death due to toxic effects nor any remarkable changes in general signs observed during the study. 


\subsection{Pharmacokinetic and biodistribution studies}

The pharmacokinetic and biodistribution of Taxol and C22-PX NPs were studied in 4T1 tumor-bearing mice. Four- to six-week-old BALB/c female mice were subcutaneously injected with $1 \times 10^{6} 4 \mathrm{~T} 1$ cells into the right flank. When tumor sizes reached 200-300 $\mathrm{mm}^{3}$, the mice were injected via tail vein with Taxol or C22-PX NPs as a single bolus. A

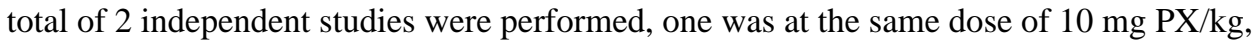
and the other one was at their MTDs (25 mg PX/kg for Taxol, and $160 \mathrm{mg}$ PX/kg for C22PX NPs). In each study, mice were sacrificed at predetermined time points (3 mice/time point), and plasma, the tissues of tumor, kidney, lung, heart, liver, and spleen were harvested. Tissues were flash-frozen in liquid nitrogen and stored at $-80^{\circ} \mathrm{C}$. Upon analysis, the frozen tissues were homogenized by Omni Bead Ruptor 24 Homogenizer (Kennesaw, GA, USA) and the drug was extracted by a mixture of methanol/acetonitrile $(2: 1, \mathrm{v} / \mathrm{v})$ (except for PX plasma samples, which were extracted by solid phase extraction method described above). The plasma concentration versus time data of both Taxol and C22-PX NPs were analyzed by noncompartmental analysis using WinNonlin (Version 5.3, Pharsight Co., Mountain View, CA, USA).

\subsection{In-vivo antitumor efficacy studies at MTD}

In-vivo antitumor efficacy studies of Taxol, C22-PX NPs, and C22-PX micelles at MTD were carried out in a 4T1 subcutaneous mouse model. Four- to six-week-old BALB/c female mice from Charles River Laboratories were subcutaneously injected with $1 \times 10^{6} 4 \mathrm{~T} 1$ cells on Day (-5), and injected via tail vein with different formulations starting from Day 0 with the treatment schedule of Q4d $\times 4$. Mice were divided into a total of 6 groups in the study (9-10 mice/group): $25 \mathrm{mg} \mathrm{PX/kg} \mathrm{of} \mathrm{Taxol,} 25$ and $160 \mathrm{mg}$ PX/kg of C22-PX NPs, 25 and $100 \mathrm{mg}$ PX/kg of C22-PX micelles, and an untreated group as the control. The mice weight and tumor size were measured every other day during the study, and tumor volume was calculated as: $\left[1 / 2 \times(\text { tumor width })^{2} \times\right.$ (tumor length) $]$. All the mice were sacrificed on Day 18 and upon sacrifice all tumors were harvested and weighed.

\subsection{Statistical analysis}

Statistical comparisons were analyzed using unpaired Student's t-test for two groups, and one-way analysis of variance (ANOVA) for multiple groups (Prism, Version 5.01, GraphPad Software Inc, La Jolla, CA, USA). Differences were considered statistically significant if the $\mathrm{p}$ value was $<0.05$.

\section{Results}

\subsection{Synthesis and characterization of C22-PX}

To increase the retention of PX in the lipid core of the lipid-based NPs, a novel fatty acidPX derivative of 2'-behenoyl-PX conjugate (C22-PX) was synthesized via the coupling of 2'-behenoyl to the C-2' hydroxyl position of PX (Figure 1). The structure was confirmed by ${ }^{1} \mathrm{H}$ - and ${ }^{13} \mathrm{C}-\mathrm{NMR}$, MS, TLC and HPLC analyses. The product yield was $85 \%$ and the purity was $>95 \%$ determined by HPLC analysis.

\subsection{Solubility of PX and C22-PX in different Miglyols}

The solubilities of PX and C22-PX in different Miglyols at room temperature were determined. Due to the introduction of a long carbon chain (C22), the solubility of C22-PX in Miglyols increased to 15-25-fold compared to that of PX (51-73 versus 3.2-3.5 $\mu \mathrm{g} / \mathrm{mg}$ Miglyol for C22-PX and PX, respectively) as shown in Figure 2. Among all the tested Miglyols, the solubility of C22-PX in Miglyol 812 was the highest $(72.5 \pm 17.1 \mu \mathrm{g} / \mathrm{mg}$ Miglyol). Therefore, Miglyol 812 was chosen as the oil phase for further C22-PX NP 
preparation. It should be noted that because all of the tested Miglyols are composed of medium chain triglycerides (C6-C10), there was no significant solubility difference within the Miglyols for both C22-PX ( $\mathrm{p}=0.1878)$ and PX ( $\mathrm{p}=0.5083)$.

\subsection{Tubulin polymerization activity of PX and C22-PX}

In general, under standard (control) reaction conditions the microtubule polymerization curve has three phases: nucleation (Figure 3A-I), growth (Figure 3A-II), and steady state (Figure 3A-III). The $\mathrm{V}_{\max }$ value (mOD/min) is used to determine the ability of a ligand (PX or C22-PX) to stabilize tubulin since this value is the most sensitive indicator of tubulin/ ligand interaction.

As shown in Figure 3B, PX at the concentration of $10 \mu \mathrm{M}$ eliminated the nucleation phase and had the greatest $\mathrm{V}_{\max }$ value of $45.0 \pm 3.6 \mathrm{mOD} / \mathrm{min}$, while the standard control had the lowest $\mathrm{V}_{\max }$ value of $25.3 \pm 3.2 \mathrm{mOD} / \mathrm{min}$. For $\mathrm{C} 22-\mathrm{PX}$ conjugate, the interaction between $\mathrm{C} 22-\mathrm{PX}$ and tubulin was dose dependent and at lower concentrations of 5 and $10 \mu \mathrm{M}, \mathrm{C} 22-$ PX had no effect on tubulin polymerization ( $\mathrm{p}>0.05$ compared to control); however, it had some tubulin polymerization activity at higher concentration of $20 \mu \mathrm{M}(\mathrm{p}<0.01$ compared to control). It should be noted that PX at $10 \mu \mathrm{M}$ was much more active than $\mathrm{C} 22-\mathrm{PX}$ at 20 $\mu \mathrm{M}(\mathrm{p}<0.01)$.

\subsection{In-vitro cytotoxicity of PX and C22-PX}

In-vitro cytotoxicity studies of free C22-PX and PX were conducted in the 4T1 cell line. The $\mathrm{IC}_{50}$ value C22-PX was about 8 -fold higher than PX $(282 \pm 42$ versus $38 \pm 1 \mathrm{nM})$. The results were expected and consistent with other reported 2'-fatty acid-PX conjugates [17, 20, 29] where the conjugates were much less toxic compared to PX since C-2' hydroxyl group is essential for PX cytotoxicity and microtubule assembly activity.

\subsection{Optimization and characterization of C22-PX NPs}

C22-PX NP formulations were engineered using a microemulsion precursor method previously described by the Mumper group [27]. The C22-PX BTM NPs were optimized in terms of drug entrapment in NPs, particle size and NP stability. Since C22-PX exhibits some surfactant property due to the presence of both lipophilic and hydrophilic moieties within the structure, C22-PX BTM NP formulations were optimized by decreasing the amount of surfactants of Brij 78 and TPGS based on our previous PX BTM NPs. A total of four BTM NP formulations (Formulations I-IV in Table 1) were investigated, and the particle sizes and drug entrapment in NPs were determined. All formulations were acceptable in terms of particle size except for Formulation I. As shown in Table 1, as the amount of surfactant utilized decreased, the amount of drug retained in the NP fraction increased. This was expected because the Miglyol and surfactants in the system competitively solubilize C22PX. Although Formulation IV led to the highest drug content in NP fractions, the NPs precipitated within one week upon storage at $4^{\circ} \mathrm{C}$. In contrast, Formulation III was much more stable and its blank NPs were stable for at least 4 months upon storage even at room temperature. Therefore, Formulation III was selected as the optimized C22-PX BTM formulation.

Interestingly, although C22-PX NPs were monodisperse with low polydispersity indices by photon correlation spectroscopy, two distinct particle size populations (100-200 nm and $\sim 50$ $\mathrm{nm}$ ) were elucidated when C22-PX NPs were passed through a Sepharose CL-4B column using PBS as the elution solvent (Figure 4A). As expected, drug content of C22-PX NPs in NP fractions 5-8 was much higher than PX NPs, which is due to the more lipophilic property of C22-PX conjugate (Figure 4B). Therefore, C22-PX micelles with particle size of $\sim 50 \mathrm{~nm}$ were used as the control. C22-PX from C22-PX micelle formulation eluted in the 
later fractions 9-14, which confirmed the existence of a population of smaller size C22-PX NPs after PBS elution (Figure 4C). It should be noted that for fractions 9-14 of C22-PX NPs with particle size of $\sim 50 \mathrm{~nm}$, it is unknown whether they are micelles, smaller oil-filled NPs or a combination of both.

\subsection{Stability of various C22-PX formulations in PBS at $37^{\circ} \mathrm{C}$}

The in-vitro stability of free C22-PX, C22-PX NPs, and C22-PX micelles in PBS was investigated. Free PX was used as the control. As shown in Figure 5A, free C22-PX underwent degradation upon incubation in $\mathrm{PBS}$ at $37^{\circ} \mathrm{C}$ as a function of time and only $\sim 30 \%$ was retained after $48 \mathrm{~h}$. It should be noted that the degradation behavior of C22-PX in PBS was very similar to free PX. In contrast, C22-PX NP or micelle formulations were very stable with no C22-PX degradation occurring within $48 \mathrm{~h}$ incubation in PBS, which indicated that the NP or micelle formulations could protect C22-PX from chemical degradation in PBS.

\subsection{C22-PX conversion to PX in tumor homogenates}

In-vitro C22-PX hydrolysis studies were performed in fresh 4T1 tumor homogenates at $37^{\circ} \mathrm{C}$ to test $\mathrm{C} 22-\mathrm{PX}$ conversion to the active form of PX. As shown in Figure 5B, a small amount of PX was formed from either free C22-PX or C22-PX NPs within the first $10 \mathrm{~h}$. After $10 \mathrm{~h}$, PX was gradually detected, and at $48 \mathrm{~h}$ the amount of PX derived from free C22PX or C22-PX NPs was 5-7\% of the total C22-PX added. This suggested that a fraction of PX could be converted from C22-PX or from its NP formulations in the tumors.

\subsection{Maximum tolerated dose (MTD) studies of C22-PX NPs}

MTD studies of Taxol, C22-PX NPs, and C22-PX micelles were conducted in 4T1 tumorbearing $\mathrm{BALB} / \mathrm{c}$ mice with the treatment schedule of $\mathrm{Q} 4 \mathrm{~d} \times 3$. The results are summarized in Table 2. The body weight loss in all groups was less than $6 \%$ throughout the whole study. Due to toxicity, 2 of 2 mice that received $40 \mathrm{mg}$ PX/kg Taxol died immediately upon the first injection, and 1 out of 3 mice died after the second treatment with C22-PX NPs and

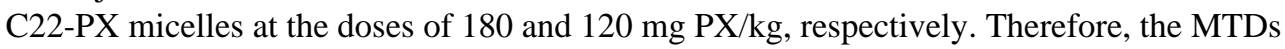
of Taxol, C22-PX NPs, and C22-PX micelles were determined as 30-40, 135-180, 90-120 $\mathrm{mg}$ PX/kg, respectively. It was noted that all mice in the Taxol group at a dose of $30 \mathrm{mg}$ PX/ $\mathrm{kg}$ lost consciousness immediately upon injection but recovered after several minutes, whereas mice treated with NP and micelle formulations did not. On the basis of the results, MTDs of 25, 160, and $100 \mathrm{mg}$ PX/kg for Taxol, C22-PX NPs, and C22-PX micelles, respectively, were chosen for subsequent in-vivo antitumor efficacy studies.

\subsection{Pharmacokinetic and biodistribution studies}

Pharmacokinetic and biodistribution studies of C22-PX NPs and Taxol at two dose levels of $10 \mathrm{mg} \mathrm{PX} / \mathrm{kg}$ and their MTDs were conducted (Figures 6 and 7). As shown in Figures 6A, 7A and Table 3, PX concentration from Taxol dropped rapidly in plasma due to its short half-life and its concentration was lower than the HPLC detection limit after $8 \mathrm{~h}$ and $48 \mathrm{~h}$ at doses of 10 and $25 \mathrm{mg} \mathrm{PX} / \mathrm{kg}$, respectively. In contrast, the concentrations of C22-PX and PX derived from C22-PX NPs declined much slower. The plasma AUCs of C22-PX from C22-PX NPs were 22- and 186-fold greater than PX from Taxol at doses of $10 \mathrm{mg}$ PX/kg and MTD, respectively. The half-life of C22-PX from C22-PX NPs increased 8-fold (8.57 h versus $1.09 \mathrm{~h}$ ) compared to PX from Taxol at a dose of $10 \mathrm{mg}$ PX/kg. The clearance rate and volume distribution of C22-PX from C22-PX NPs in plasma were also significantly decreased. All these results suggested that C22-PX NPs had a long circulation time and were largely confined in the blood stream. The distributions of C22-PX from C22-PX NPs and PX from Taxol in various tissues are summarized in Figures 6B, 6C, 7B and 7C. In general, 
PX concentration from Taxol decreased as a function of time and PX was widely distributed into most tissues including liver, lung, kidney and heart. In contrast, C22-PX from C22-PX NPs reached a maximal concentration between $1-4 \mathrm{~h}$ after intravenous administration in most tissues, and C22-PX was mainly distributed in the liver and spleen. Importantly, tumor AUCs of C22-PX from NP formulations were 2.6- and 24.4-fold higher than PX from Taxol at doses of $10 \mathrm{mg}$ PX/kg and MTD, respectively. Tumor AUC of PX derived from C22-PX NPs was 1.5-fold greater than PX from Taxol at their MTDs (Figure 7D and Table 3).

\subsection{In-vivo antitumor efficacy studies at MTD}

In-vivo antitumor efficacy studies of Taxol, C22-PX NPs and C22-PX micelles at MTD were evaluated in a 4T1 subcutaneous mouse model (Figure 8). Two dose levels of C22-PX NPs and micelles were applied in the study: MTD of Taxol at $25 \mathrm{mg} \mathrm{PX} / \mathrm{kg}$, and their unique MTDs as determined (160 mg PX/kg for C22-PX NPs and $100 \mathrm{mg}$ PX/kg for C22PX micelles). Treatments with all formulations at any dose level were well tolerated by the mice since their body weight was comparable to the untreated mice (Figure 8A), indicating no induced toxicity by either NP or micelle formulations even at their MTDs. As expected, the tumor growth rate of untreated group was the highest. The C22-PX NPs at MTD exhibited significantly more potent in-vivo tumor inhibitory activities than other groups starting from Day 6 (Figure 8B). It should be noted that both C22-PX NPs and C22-PX micelles had similar antitumor activity with Taxol at the dose of $25 \mathrm{mg} \mathrm{PX} / \mathrm{kg}$, and the increase from 25 to $100 \mathrm{mg}$ PX/kg of C22-PX micelles did not further improve the antitumor efficacy ( $p>0.05$ ). Results from tumor weight (Figure 8C) were consistent with tumor size measurements, where tumors from the untreated mice were the largest and heaviest, and tumors from mice treated with C22-PX NPs at MTD were the smallest and statistically significant compared to all other groups $(\mathrm{p}<0.05)$.

\section{Discussion}

PX is a very effective anticancer drug; however, due to its low water solubility and lack of ionizable functional groups, a great deal of effort has been focused to synthesize more water-soluble PX derivatives [15]. Alternatively, more lipophilic PX derivatives have been developed for lipid-based formulations. PX derivatives are generally designed at the C-2' position because the free hydroxyl group at this position is much more reactive than the $\mathrm{C}-7$ hydroxyl group, and PX-ester based derivatives can be synthesized selectively at C-2' group of PX without protecting the C-7 hydroxyl group. To date, several synthesized 2'-fatty acidPX ester derivatives have been reported, such as 2'-linoleic acid-PX [30], 2'carbonylcholesterol-PX [20], 2'-oleate-PX [17], 2'-a-bromohexadecanoyl-PX [29], and DHA-PX [19, 23], all of which showed some benefits compared to Taxol in-vitro and/or invivo. For example, the lipophilic 2'-oleate-PX conjugate showed significantly greater AUC, higher $\mathrm{C}_{\max }$, and lower systemic clearance compared to PX formulated in Cremophor EL/ Ethanol in a rabbit model [17]. Perkins et al. synthesized 2'-a-bromohexadecanoyl-PX conjugate and further incorporated it into lipid-coated particles termed "lipocores". Their invivo studies demonstrated that the lipocores were much less toxic than Taxol after i.p or i.v. administration and were active in the ovarian carcinoma SCID mice model [29]. As mentioned above, DHA-PX has also been investigated. In an M109 s.c. mouse model, the tumor AUCs of PX from DHA-PX conjugate were 1.71- and 5.79-fold greater than Taxol when i.v. injected into mice at equimolar and equitoxic doses, respectively [19]. However, results from the most recent Phase III study to treat metastatic malignant melanoma were disappointing where DHA-PX was not superior to dacarbazine in terms of overall survival, and there were no significant differences between the two drugs in patient response rate, duration of response, and time to progression on an every 3-week treatment schedule [22]. 
In this study, a novel 2'-behenoyl-PX ester derivative (C22-PX) was successfully synthesized and its structure was confirmed by ${ }^{1} \mathrm{H}-$ and ${ }^{13} \mathrm{C}-\mathrm{NMR}$, MS, TLC and HPLC analyses. Since the C-2' hydroxyl group is essential for PX cytotoxicity and microtubule assembly activity, the C22-PX conjugate should be much less toxic compared to PX if it does not convert to its more active form of PX. This was supported by 1) the tubulin polymerization assay (Figure 3). PX could stabilize microtubule proteins at $10 \mu \mathrm{M}$, however, C22-PX had no microtubule activity at this concentration but did have a dose dependent ability to stabilize microtubules. The results were similar to DHA-PX and 2'-linoleic acidPX where both of the prodrugs were not able to assemble microtubule at the concentration level of $10 \mu \mathrm{M}[19,30]$; 2) in-vitro cytotoxicity studies in $4 \mathrm{~T} 1$ cells showed that the cytotoxicity of C22-PX was much lower than its parent $\mathrm{PX}\left(\mathrm{IC}_{50}\right.$ : $282 \pm 42$ versus $38 \pm 1$ $\mathrm{nM})$ after $48 \mathrm{~h}$ incubation in cell culture medium. These results were consistent with other 2'-fatty acid-PX prodrugs. For example, 2'-a-bromohexadecanoyl-PX, and 2'-diglycolateand succinate-PX produgs were 70- and 10-100-fold, respectively, less potent compared to $\mathrm{PX}$ against human MCF-7 cells at a $72 \mathrm{~h}$ incubation time [18, 29]. DHA-PX was reported to be cytotoxic at the $\mu \mathrm{M}$ concentration range, while PX was in $\mathrm{nM}$ range for a variety of human tumor cell lines [19]. Similarly, the cytotoxicity of 2'-oleate-PX in lipid emulsions was much lower than that of PX but with time dependent manner, where the $\mathrm{IC}_{50}$ values were 5500, 500, 150, and $100 \mathrm{nM}$ for 24, 48, 72, and $96 \mathrm{~h}$ incubation, respectively, compared to an $\mathrm{IC}_{50}$ of $50 \mathrm{nM}$ for Taxol at $48 \mathrm{~h}$ in HeLa cells [17]. The differences in the $\mathrm{IC}_{50}$ between C22-PX conjugate and PX may be due to a slow hydrolysis rate of C22-PX to release PX, which was supported by C22-PX hydrolysis in 4T1 tumor homogenates, where only about 5-7\% of C22-PX was converted to PX during $48 \mathrm{~h}$ incubation for both free C22PX and C22-PX NPs (Figure 5B). To provide faster hydrolysis rates, electron-withdrawing substituents in the a-position of the fatty acid can be introduced. A 2'- $\mathrm{a}-$ bromohexadecanoyl-PX has been synthesized and is currently under investigation in our laboratory.

The C22-PX BTM NP formulations were engineered using a microemulsion precursor method previously described by the Mumper group [27, 31]. Miglyol 812 was used as the oil phase based on its highest solubilization capacity for C22-PX (Figure 2), and because C22PX solubility in the oil phase of Miglyol 812 increased 25-fold compared to PX (72.5 \pm 17.1 versus $3.2 \pm 0.1 \mu \mathrm{g} / \mathrm{mg}$ Miglyol), the drug entrapment in our lipid-based NPs improved significantly which was confirmed by GPC. When C22-PX NP formulations passed through a Sepharose CL-4B GPC column, 47\% of the drug conjugate was retained in the $200 \mathrm{~nm}$ NPs versus only $10 \%$ for previous PX NPs (Figure 4B), likely due to the more lipophilic property of C22-PX. Interestingly, although C22-PX NPs showed a mono-distribution profile with a low polydispersity index by photon correlation spectroscopy, two distinct particle size populations were elucidated by the GPC method (Figure 4A). These results were consistent with previous findings reported by Jay et al. on solid lipid NPs containing stearyl alcohol where two distinct size populations were obtained following elution through a GPC column [28]. Previously, an ultracentrifuge method was applied to determine the drug entrapment efficiency and the results may be somehow misleading since drugs may precipitate and aggregate during the ultracentrifugation process and/or may also bind to the filter membrane. In contrast, the GPC method is more dynamic and presumably more accurate in predicting the in-vivo fate of NPs.

The in-vitro stability of free C22-PX and C22-PX NPs was investigated at $37^{\circ} \mathrm{C}$ in PBS (Figure 5A). Interestingly, C22-PX degraded in PBS over time and only $30 \%$ of C22-PX was retained after $48 \mathrm{~h}$ incubation. It was reported that PX hydrolyzes to its main degradation products of baccatin III and V, 10-deacetylbaccatin III, and 7-epi-PX in PBS, and its degradation kinetics was determined to be first order for the first $12 \mathrm{~h}$ [32]. In the present studies, free PX was used as the control and the degradation behavior of C22-PX in 
PBS was found to be very similar to that of free PX. Therefore, it was hypothesized that C22-PX and PX undergo similar degradation pathways in PBS. In contrast, both C22-PX NP and micelle formulations were stable and no C22-PX degradation occurred during a $48 \mathrm{~h}$ incubation in PBS, which indicated that the NP or micelle formulations could protect C22PX from degradation in PBS.

Since C22-PX BTM NPs were free of Cremophor EL and ethanol which are the solvents in Taxol, the vehicle associated toxicity should be much less. In fact, this was confirmed by the 6-fold greater MTD of C22-PX NPs compared to Taxol (Table 2). The antitumor efficacy of C22-PX NPs at MTD was then evaluated in a 4T1 s.c. mouse model with a Q4d $\times 4$ treatment schedule. C22-PX NPs at MTD exhibited no toxicity based on the body weight change which was comparable to the untreated mice (Figure 8A). More importantly, C22PX NPs demonstrated a significantly superior antitumor activity compared to Taxol and C22-PX micelles at MTD (Figures 8B and 8C). To better understand the in-vivo behavior of C22-PX NPs, a total of two pharmacokinetic and tissue biodistribution studies of C22-PX NPs versus Taxol were conducted at doses of $10 \mathrm{mg} P X / \mathrm{kg}$ and MTD, respectively (Figures 6-7). PX concentration from Taxol declined very rapidly in plasma while C22-PX from C22-PX NP formulations had much longer circulation time in blood, where the plasma AUCs of C22-PX from C22-PX NPs were 22- and 186-fold greater than those of PX from Taxol at doses of $10 \mathrm{mg}$ PX/kg and MTD, respectively. The prolonged circulation time would enable NP formulations to take full advantage of EPR effect thus more of the drug would be expected to accumulate at the tumor site. This was confirmed by the fact that the tumor AUCs of C22-PX from the NP formulations were 2.6- and 24.4-fold greater than that of PX from Taxol at doses of $10 \mathrm{mg}$ PX/kg and MTD, respectively. It should be noted that the tumor AUC of PX derived from C22-PX NPs was 1.5-fold greater than PX from Taxol at their MTDs. Taken together, there are at least three possible reasons for the enhanced antitumor efficacy of C22-PX NPs at MTD: 1) the 6-fold increase in MTD allowed much higher doses of the drug to be delivered to mice; 2) C22-PX NPs had a significantly improved pharmacokinetic profile and prolonged circulation time in the blood, and much higher drug accumulation in the tumor due to the EPR effect, based on the facts that the plasma and tumor AUCs of C22-PX from C22-PX NPs were 186- and 24.4-fold greater than PX from Taxol at MTD, respectively; and 3) Once C22-PX NPs reached the tumor site, PX was converted and released from C22-PX in a sustained manner.

It should be noted that the BTM NPs were able to overcome P-gp mediated MDR both invitro and in-vivo, and the mechanisms were investigated and included: 1) increase drug cell uptake and retention; 2) inhibition of P-gp; and 3) transient depletion of ATP [13]. Since resistance to PX remains a significant unmet problem, the ability of BTM NPs to address this problem may be very promising. Unfortunately, the $4 \mathrm{~T} 1$ murine cell line in this study is not resistant [33]. However, the 4T1 cell line was very aggressive based on the facts that 1) the tumor size of untreated mice reached $\sim 1500 \mathrm{~cm}^{3}$ after 20-25 days of cell injection; 2) the Q4d $\times 4$ treatment with Taxol at MTD of $25 \mathrm{mg} / \mathrm{kg}$ did not show better antitumor inhibition effect compared to untreated mice (Figure 8B). It is expected that C22-PX BTM NPs would have much better antitumor efficacy if resistant and less aggressive mouse models were to be utilized. In addition, EGFR targeted NPs could potentially be engineered to further improve the anticancer efficacy and enhance the therapeutic index of PX.

\section{Acknowledgments}

This research was supported by NIH-NCI R01 CA115197 and NIH-NCI U54 CA151652. The authors would like to thank Charlene M. Santos and her group for their efforts and help with the animal studies. 


\section{References}

1. Jordan MA, Wilson L. Microtubules as a target for anticancer drugs. Nat Rev Cancer. 2004; 4:253265. [PubMed: 15057285]

2. Gelderblom H, Verweij J, Nooter K, Sparreboom A. Cremophor EL: the drawbacks and advantages of vehicle selection for drug formulation. European Journal of Cancer. 2001; 37:1590-1598. [PubMed: 11527683]

3. Gallo JM, Li S, Guo P, Reed K, Ma J. The effect of P-glycoprotein on paclitaxel brain and brain tumor distribution in mice. Cancer Res. 2003; 63:5114-5117. [PubMed: 12941842]

4. Lo J-T, Chen B-H, Lee T-M, Han J, Li J-L. Self-emulsifying O/W formulations of paclitaxel prepared from mixed nonionic surfactants. J. Pharm. Sci. 2010; 99:2320-2332. [PubMed: 19894274]

5. Wang F, Zhang D, Zhang Q, Chen Y, Zheng D, Hao L, Duan C, Jia L, Liu G, Liu Y. Synergistic effect of folate-mediated targeting and verapamil-mediated P-gp inhibition with paclitaxel -polymer micelles to overcome multi-drug resistance. Biomaterials. 2011; 32:9444-9456. [PubMed: 21903258]

6. Yoshizawa Y, Kono Y, Ogawara K-I, Kimura T, Higaki K. PEG liposomalization of paclitaxel improved its in vivo disposition and anti-tumor efficacy. Int. J. Pharm. 2011; 412:132-141. [PubMed: 21507344]

7. Lee M-K, Lim S-J, Kim C-K. Preparation, characterization and in vitro cytotoxicity of paclitaxelloaded sterically stabilized solid lipid nanoparticles. Biomaterials. 2007; 28:2137-2146. [PubMed: 17257668]

8. Rose WC, Clark JL, Lee FYF, Casazza AM. Preclinical antitumor activity of water-soluble paclitaxel derivatives. Cancer Chemother. Pharmacol. 1997; 39:486-492. [PubMed: 9118459]

9. Grover S, Rimoldi JM, Molinero AA, Chaudhary AG, Kingston DGI, Hamel E. Differential effects of paclitaxel (Taxol) analogs modified at positions C-2, C-7, and C-3' on tubulin polymerization and polymer stabilization: identification of a hyperactive paclitaxel derivative. Biochemistry. 1995; 34:3927-3934. [PubMed: 7696257]

10. Feng X, Yuan Y-J, Wu J-C. Synthesis and evaluation of water-Soluble paclitaxel prodrugs. Bioorg. Med. Chem. Lett. 2002; 12:3301-3303. [PubMed: 12392737]

11. Gradishar WJ, Tjulandin S, Davidson N, Shaw H, Desai N, Bhar P, Hawkins M, O'Shaughnessy J. Phase III trial of nanoparticle albumin-bound paclitaxel compared with polyethylated castor oilbased paclitaxel in women with breast cancer. Journal of Clinical Oncology. 2005; 23:7794-7803. [PubMed: 16172456]

12. Dong X, Mattingly CA, Tseng M, Cho M, Adams VR, Mumper RJ. Development of new lipidbased paclitaxel nanoparticles using sequential simplex optimization. European Journal of Pharmaceutics and Biopharmaceutics. 2009; 72:9-17. [PubMed: 19111929]

13. Dong X, Mattingly CA, Tseng MT, Cho MJ, Liu Y, Adams VR, Mumper RJ. Doxorubicin and paclitaxel-loaded lipid-based nanoparticles overcome multidrug resistance by inhibiting Pglycoprotein and depleting ATP. Cancer Res. 2009; 69:3918-3926. [PubMed: 19383919]

14. Ryu B-Y, Sohn J-S, Hess M, Choi S-K, Choi J-K, Jo B-W. Synthesis and anti-cancer efficacy of rapid hydrolysed water-soluble paclitaxel pro-drugs. Journal of Biomaterials Science, Polymer Edition. 2008; 19:311-324. [PubMed: 18325233]

15. Dhanikula AB, Panchagnula R. Preparation and characterization of water-soluble prodrug, liposomes and micelles of paclitaxel. Current Drug Delivery. 2005; 2:75-91. [PubMed: 16305410]

16. Hayashi Y, Skwarczynski M, Hamada Y, Sohma Y, Kimura T, Kiso Y. A novel approach of watersoluble paclitaxel prodrug with no auxiliary and no byproduct: design and synthesis of isotaxel. J. Med. Chem. 2003; 46:3782-3784. [PubMed: 12930140]

17. Lundberg BB, Risovic V, Ramaswamy M, Wasan KM. A lipophilic paclitaxel derivative incorporated in a lipid emulsion for parenteral administration. J. Control. Release. 2003; 86:93100. [PubMed: 12490375]

18. Ansell SM, Johnstone SA, Tardi PG, Lo L, Xie S, Shu Y, Harasym TO, Harasym NL, Williams L, Bermudes D, Liboiron BD, Saad W, Prud'homme RK, Mayer LD. Modulating the therapeutic 
activity of nanoparticle delivered paclitaxel by manipulating the hydrophobicity of prodrug conjugates. J. Med. Chem. 2008; 51:3288-3296. [PubMed: 18465845]

19. Bradley MO, Webb NL, Anthony FH, Devanesan P, Witman PA, Hemamalini S, Chander MC, Baker SD, He LF, Horwitz SB, Swindell CS. Tumor targeting by covalent conjugation of a natural fatty acid to paclitaxel. Clinical Cancer Research. 2001; 7:3229-3238. [PubMed: 11595719]

20. Stevens PJ, Sekido M, Lee RJ. A folate receptor-targeted lipid nanoparticle formulation for a lipophilic paclitaxel prodrug. Pharm Res. 2004; 21:2153-2157. [PubMed: 15648245]

21. Harries M, O'Donnell A, Scurr M, Reade S, Cole C, Judson I, Greystoke A, Twelves C, Kaye S. Phase I//II study of DHA-paclitaxel in combination with carboplatin in patients with advanced malignant solid tumours. Br J Cancer. 2004; 91:1651-1655. [PubMed: 15494716]

22. Bedikian AY, DeConti RC, Conry R, Agarwala S, Papadopoulos N, Kim KB, Ernstoff M. Phase 3 study of docosahexaenoic acid-paclitaxel versus dacarbazine in patients with metastatic malignant melanoma. Ann. Oncol. 2011; 22:787-793. [PubMed: 20855467]

23. Bradley MO, Swindell CS, Anthony FH, Witman PA, Devanesan P, Webb NL, Baker SD, Wolff AC, Donehower RC. Tumor targeting by conjugation of DHA to paclitaxel. J. Control. Release. 2001; 74:233-236. [PubMed: 11489499]

24. Kuznetsova L, Chen J, Sun L, Wu XY, Pepe A, Veith JA, Pera P, Bernacki RJ, Ojima I. Syntheses and evaluation of novel fatty acid-second-generation taxoid conjugates as promising anticancer agents. Bioorg. Med. Chem. Lett. 2006; 16:974-977. [PubMed: 16298526]

25. Shelanski ML, Gaskin F, Cantor CR. Microtubule assembly in the absence of added nucleotides. Proceedings of the National Academy of Sciences. 1973; 70:765-768.

26. Lee JC, Timasheff SN. In vitro reconstitution of calf brain microtubules: effects of solution variables. Biochemistry. 1977; 16:1754-1764. [PubMed: 856260]

27. Ma P, Dong X, Swadley CL, Gupte A, Leggas M, Ledebur HC, Mumper RJ. Development of idarubicin and doxorubicin solid lipid nanoparticles to overcome Pgp-mediated multiple drug resistance in leukemia. J. Biomed. Nanotechnol. 2009; 5:151-161. [PubMed: 20055093]

28. Kim J-K, Howard MD, Dziubla TD, Rinehart JJ, Jay M, Lu X. Uniformity of drug payload and its effect on stability of solid lipid nanoparticles containing an ester prodrug. ACS Nano. 2010; 5:209-216. [PubMed: 21158414]

29. Ali S, Ahmad I, Peters A, Masters G, Minchey S, Janoff A, Mayhew E. Hydrolyzable hydrophobic taxanes: synthesis and anti-cancer activities. Anti-Cancer Drugs. 2001; 12:117-128. [PubMed: $11261884]$

30. Ke XY, Zhao BJ, Zhao X, Wang Y, Huang Y, Chen XM, Zhao BX, Zhao SS, Zhang XA, Zhang QA. The therapeutic efficacy of conjugated linoleic acid - paclitaxel on glioma in the rat. Biomaterials. 2010; 31:5855-5864. [PubMed: 20430438]

31. Feng L, Wu HL, Ma P, Mumper RJ, Benhabbour SR. Development and optimization of oil-filled lipid nanoparticles containing docetaxel conjugates designed to control the drug release rate in vitro and in vivo. Int. J. Nanomed. 2011; 6:2545-2556.

32. Dordunoo SK, Burt HM. Solubility and stability of taxol: effects of buffers and cyclodextrins. Int. J. Pharm. 1996; 133:191-201.

33. Zhao LH, Jin XQ, Xu YH, Guo YX, Liang R, Guo ZH, Chen TF, Sun YH, Ding XH. Functional study of the novel multidrug resistance gene HA117 and its comparison to multidrug resistance gene 1. J. Exp. Clin. Cancer Res. 2010; 29:98-104. [PubMed: 20642825] 

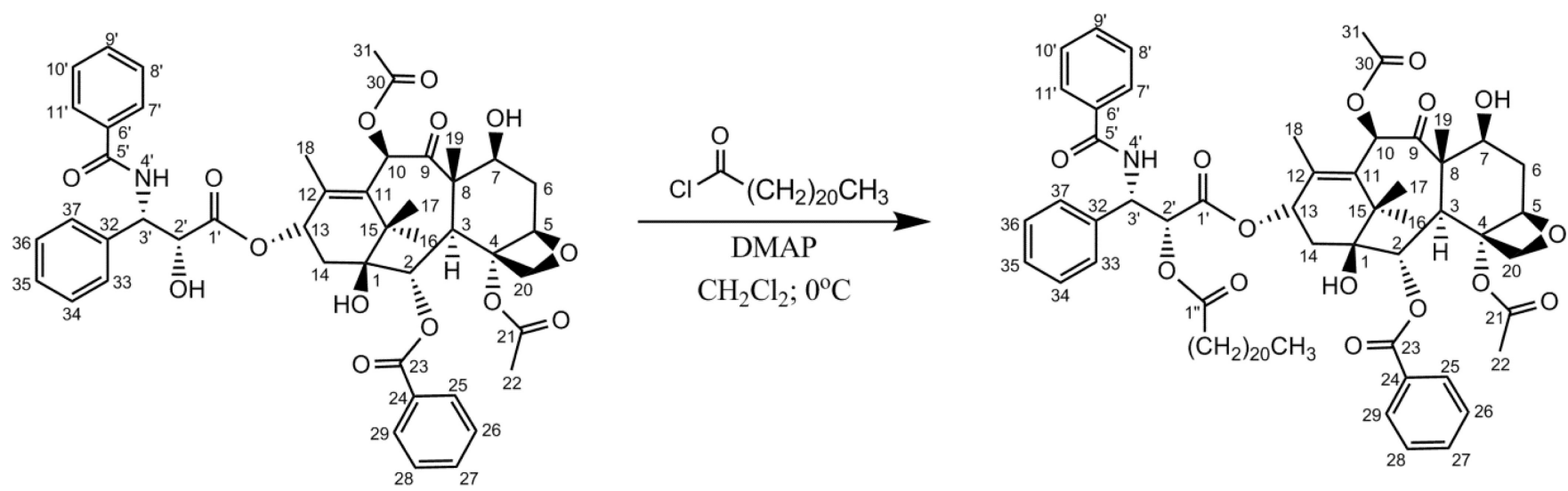

Figure 1.

Synthesis of C22-PX conjugate via a one-step esterification reaction of PX and behenoyl chloride. The synthesized C22-PX conjugate was confirmed by ${ }^{1} \mathrm{H}$ - and ${ }^{13} \mathrm{C}-\mathrm{NMR}$, MS, TLC, and HPLC analyses. 


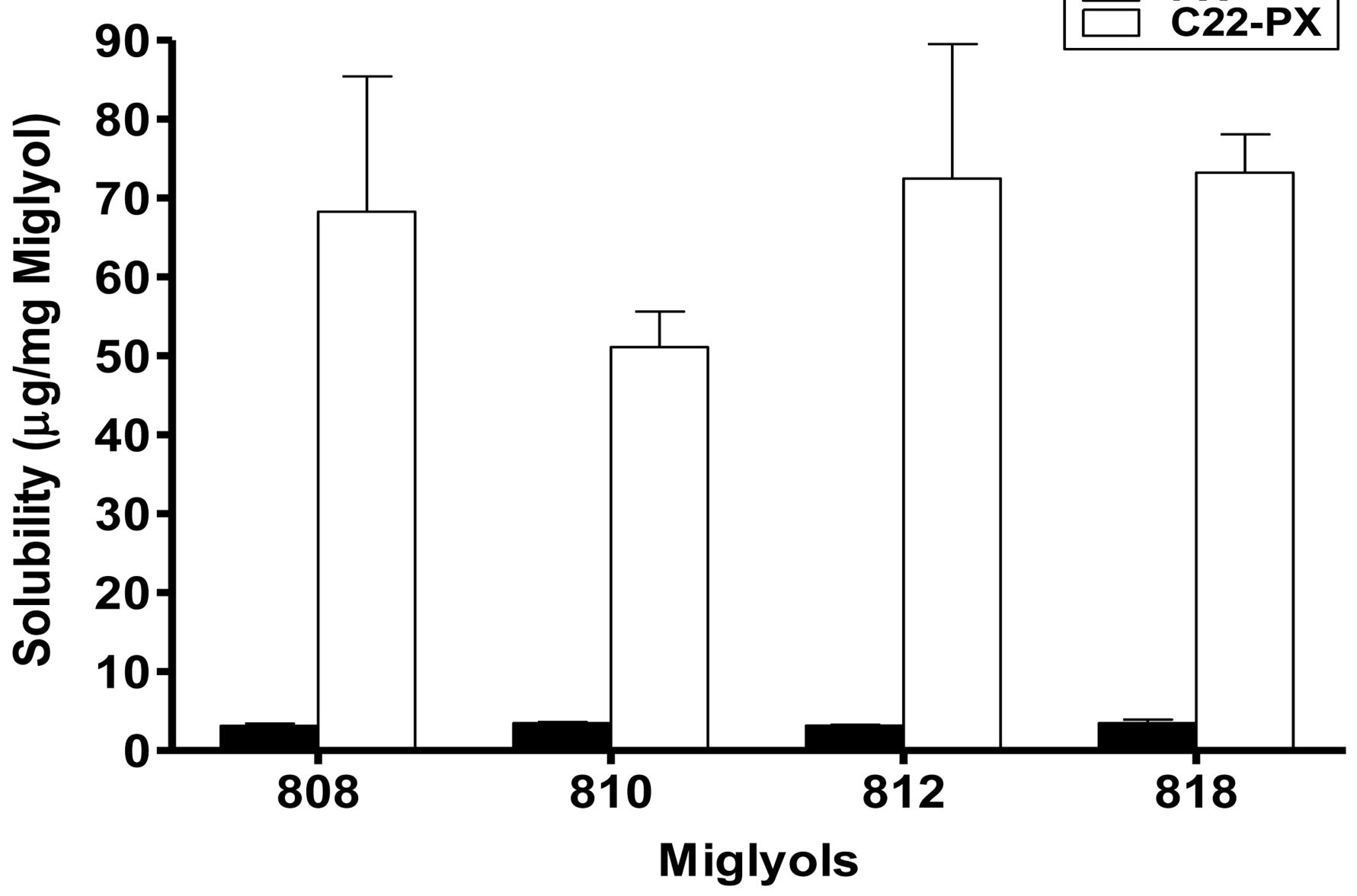

Figure 2.

Solubility of C22-PX conjugate and PX in different Miglyols. Excess C22-PX and PX were added into Miglyols and were kept stirred for $24 \mathrm{~h}$ at room temperature. The samples were then centrifuged at $14,000 \mathrm{~g}$ for $20 \mathrm{~min}$ at $25^{\circ} \mathrm{C}$. After that, the supernatant was collected, accurately weighed, and dissolved in methanol in a $50 \mathrm{ml}$ volumetric flask. The concentrations of C22-PX and PX were determined by HPLC and LC-MS analysis, respectively. Results are expressed as mean $\pm \mathrm{SD}(\mathrm{n}=3)$. 
(A)

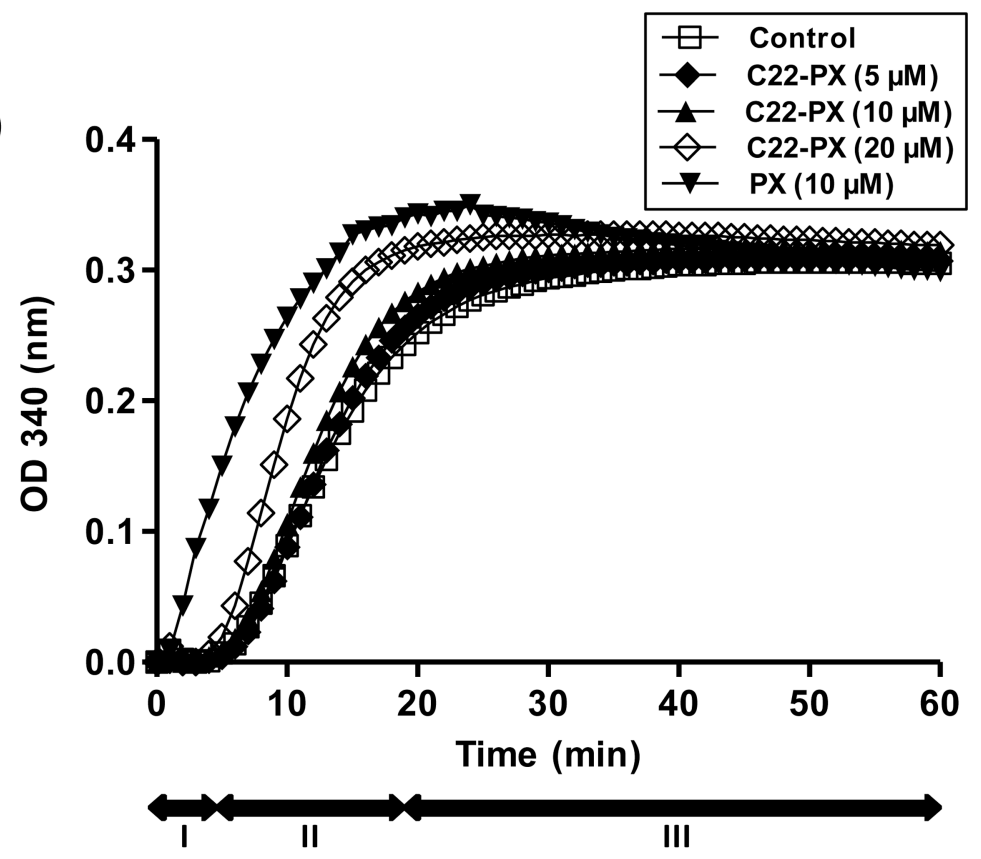

(B)

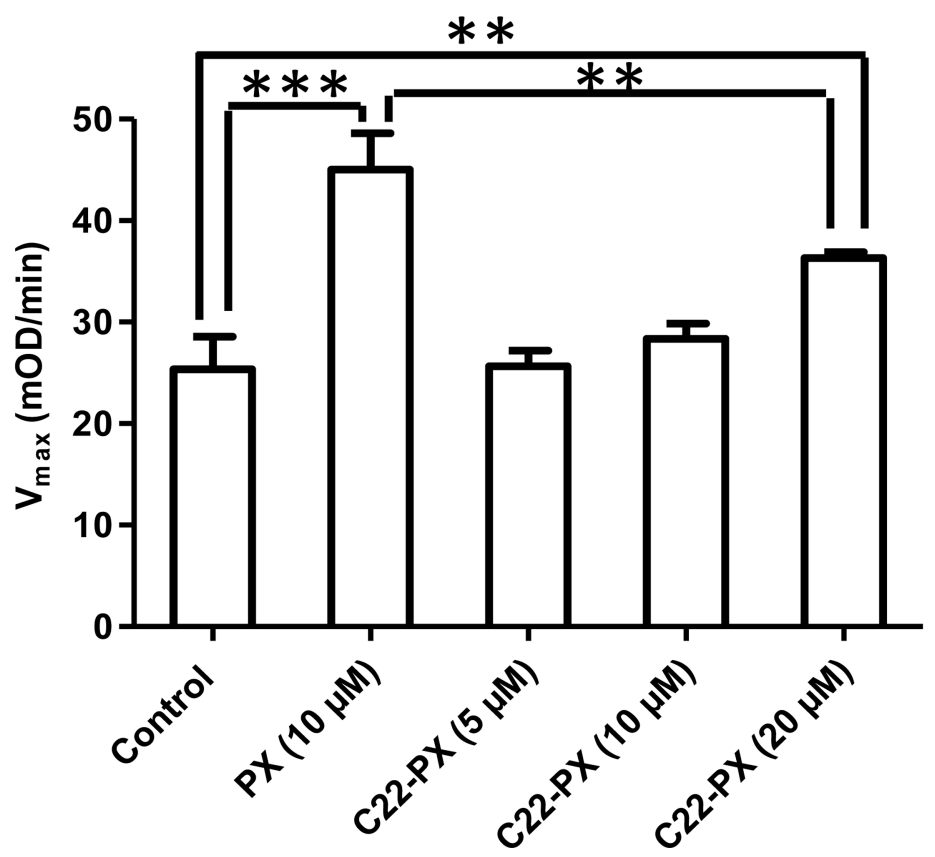

Figure 3.

Tubulin polymerization assay of C22-PX conjugate. The standard (control) polymerization reaction contains $100 \mu \mathrm{l}$ of $3 \mathrm{mg} / \mathrm{mL}$ tubulin in $80 \mathrm{mM}$ PIPES pH 6.9, $0.5 \mathrm{mM}$ EGTA, 2 $\mathrm{mM} \mathrm{MgCl} 2,1 \mathrm{mM} \mathrm{GTP}$, and $10 \%$ glycerol. The assay was conducted at $37^{\circ} \mathrm{C}$ and the tubulin polymerization was monitored by the change of the absorbance at $340 \mathrm{~nm}$ over a 60 min period (1 reading per min) with a temperature controlled Microplate Reader. C22-PX concentrations of 5,10 , and $20 \mu \mathrm{M}$ were applied in the assay. PX $(10 \mu \mathrm{M})$ was used as the positive control. (A) Tubulin polymerization curves of control, PX, and C22-PX. (B) $\mathrm{V}_{\max }$ values of control, PX, and C22-PX. Data are presented as mean $\pm \mathrm{SD}(\mathrm{n}=3)$. (** $\mathrm{p}<0.01$, $* * * \mathrm{p}<0.001)$ 


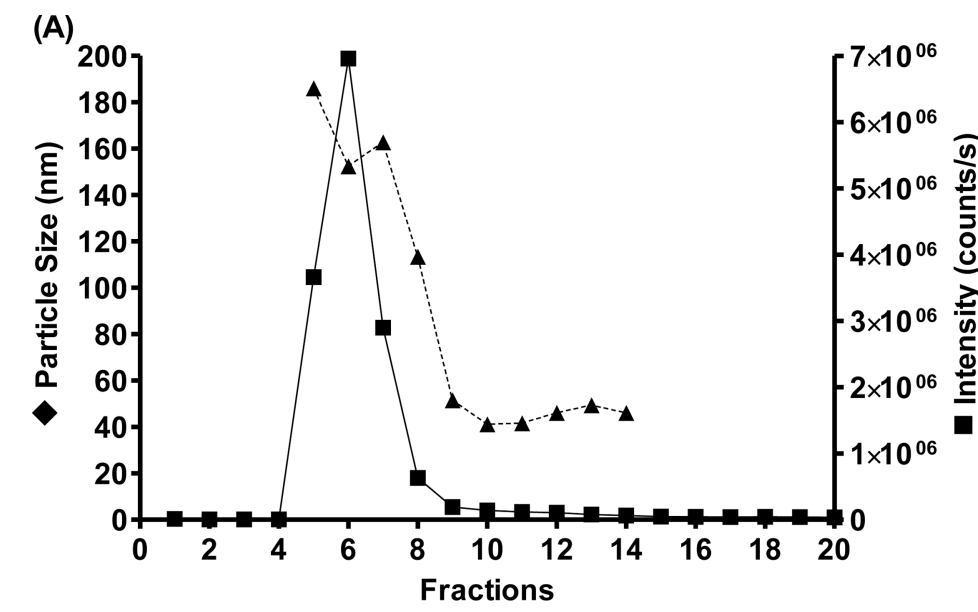

(B)
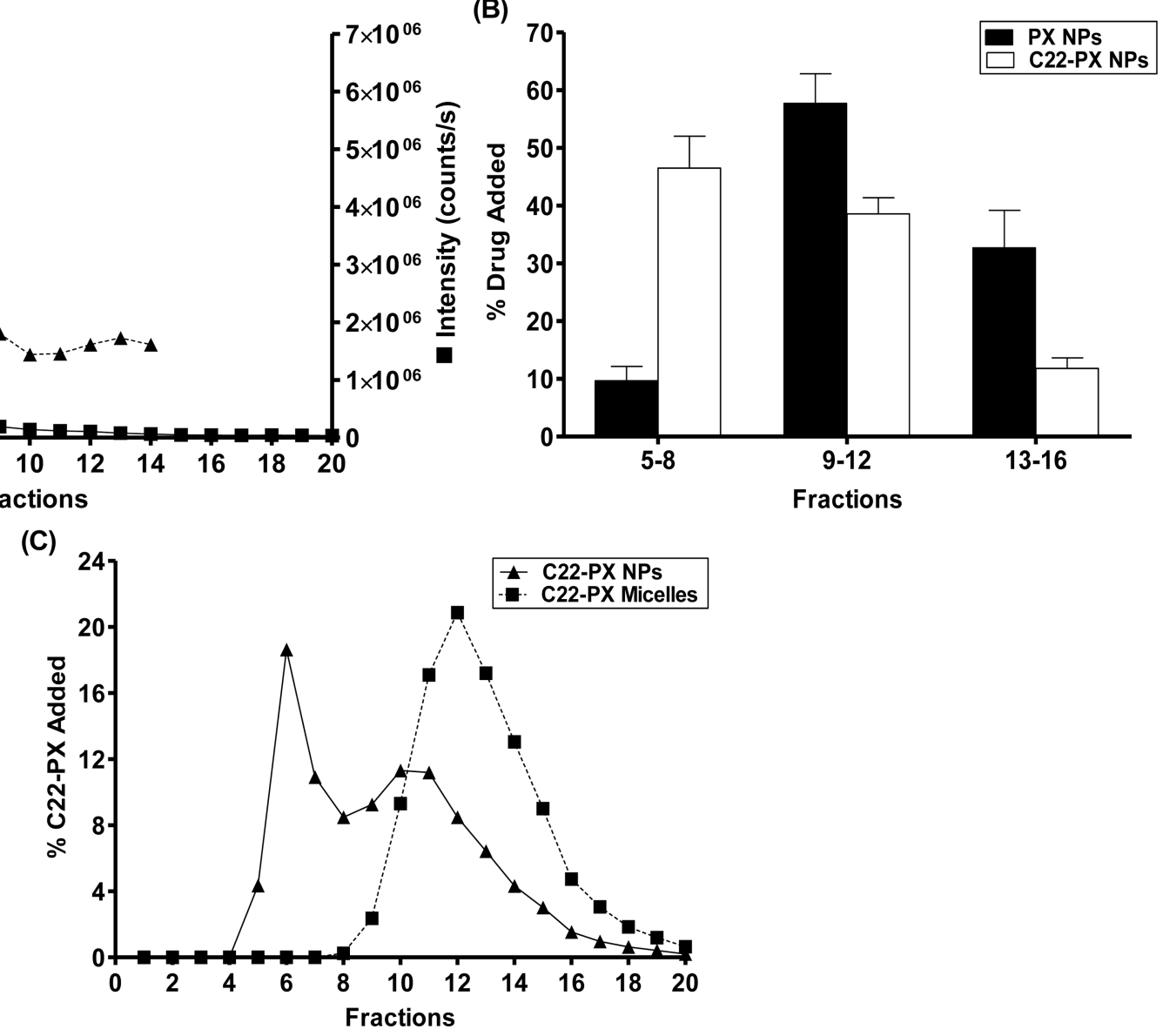

Figure 4.

Gel permeation chromatography elution profiles with a Sepharose CL-4B column. A total of 20 fractions were collected (1 ml/fraction) with the elution solvent of PBS. (A) Particle size $(\downarrow)$ and intensity ( $\mathbf{\square})$ of each fraction of C22-PX NPs. (B) \% drug retention in various fractions of PX NPs $(\square)$ and C22-PX NPs ( $\square$ ). Results are expressed as mean \pm SD (n=3). (C) \% C22-PX retention in each fraction of C22-PX NPs $(\mathbf{\square})$ or C22-PX micelles (O). \% drug retention was calculated as: [(mass of drug in each fraction) / (total mass of drug loaded into the column $)] \times 100 \%(\mathrm{w} / \mathrm{w})$ 
(A)

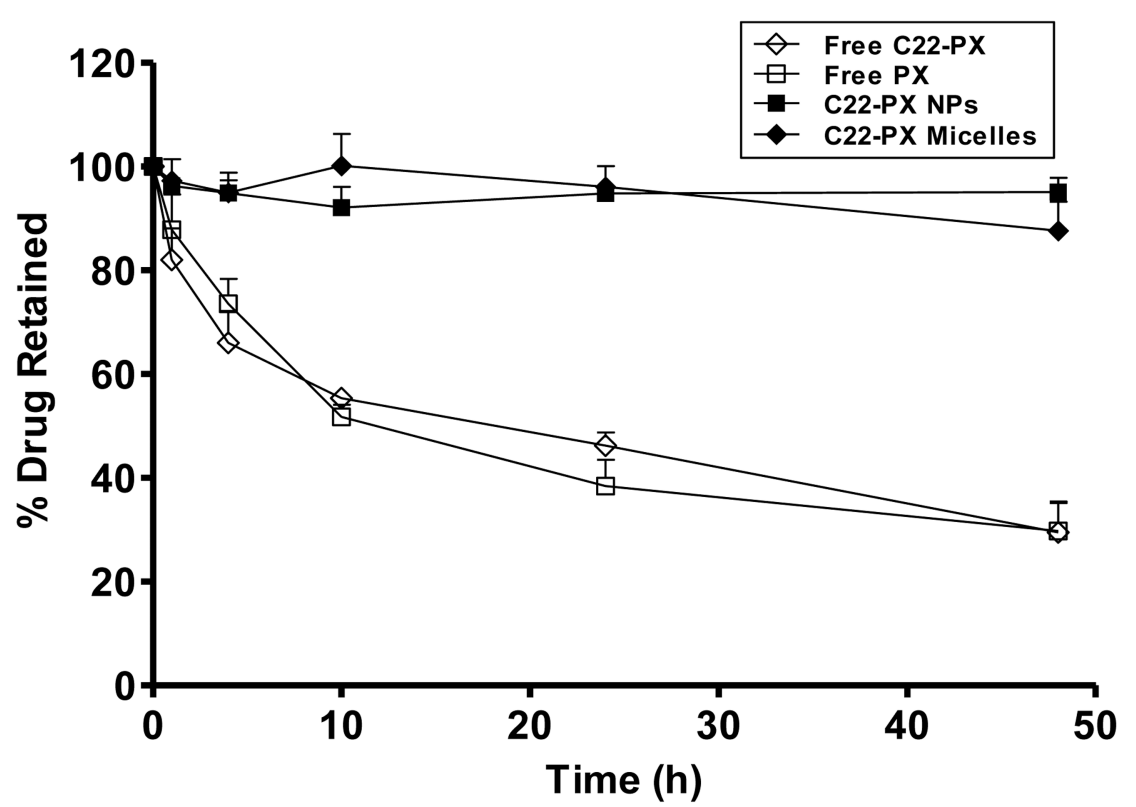

(B)

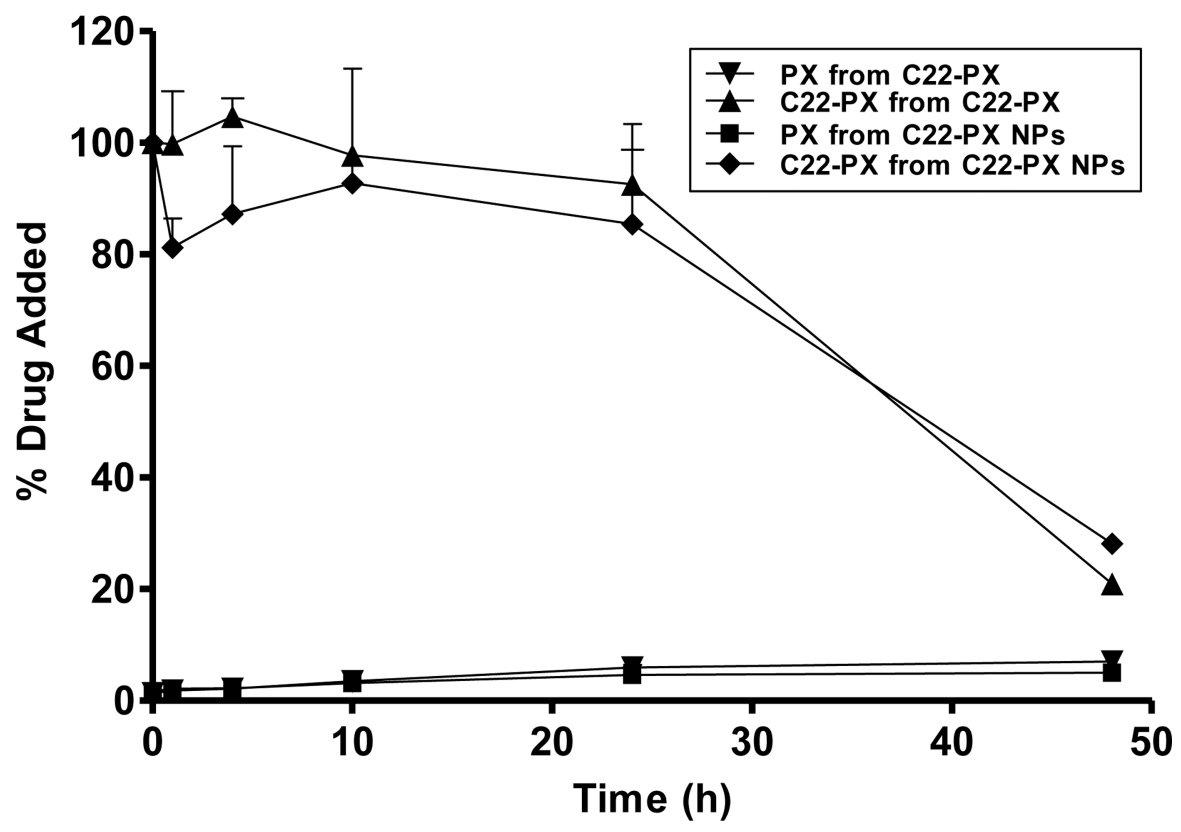

Figure 5.

Various C22-PX formulations incubated in PBS and $4 \mathrm{~T} 1$ tumor homogenates at $37^{\circ} \mathrm{C}$ in a water bath. (A) Different C22-PX formulations incubated in PBS and at the predetermined time points, samples were collected and the concentration of C22-PX were determined by HPLC. Free PX was used as the control and the final concentrations of the drug in the experiment were $10 \mu \mathrm{g} / \mathrm{ml}$. (B) C22-PX and C22-PX NPs were spiked into fresh 4T1 tumor homogenates at the concentration of $10 \mu \mathrm{g} / \mathrm{g}$ tumor and incubated at $37^{\circ} \mathrm{C}$ in a water bath. At predetermined time points, $\sim 40-50 \mathrm{mg}$ of samples was taken out. The concentrations of both C22-PX and PX converted from C22-PX or C22-PX NPs were determined by HPLC or LCMS analysis. Results are expressed as mean $\pm \mathrm{SD}(\mathrm{n}=3)$. 
(A)

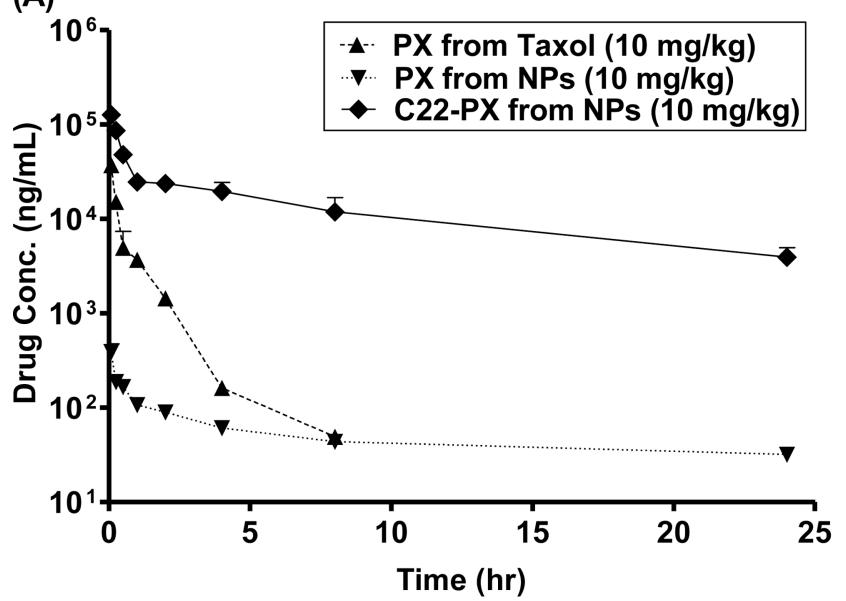

(C)

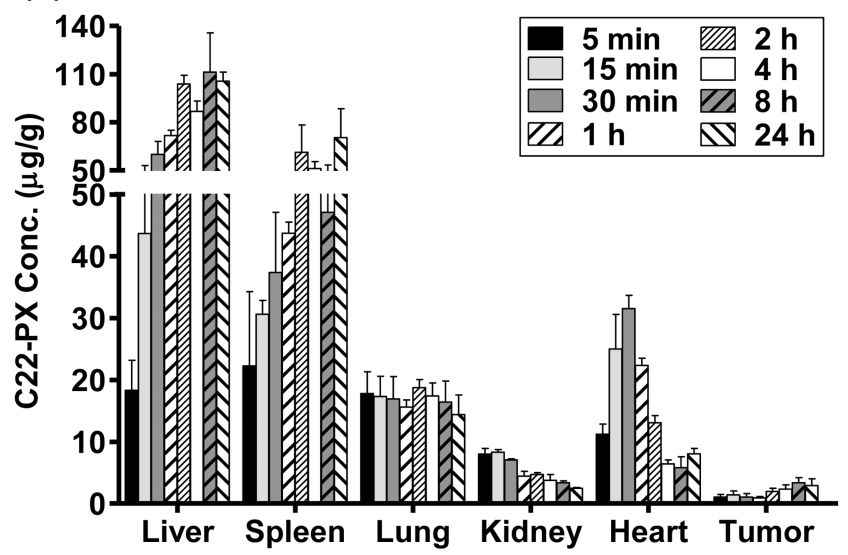

(B)

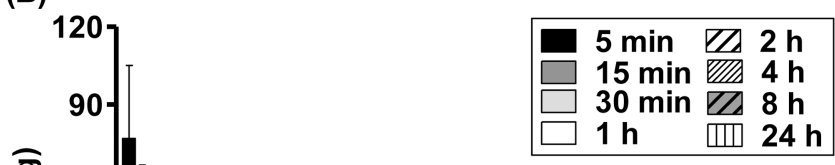

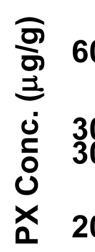

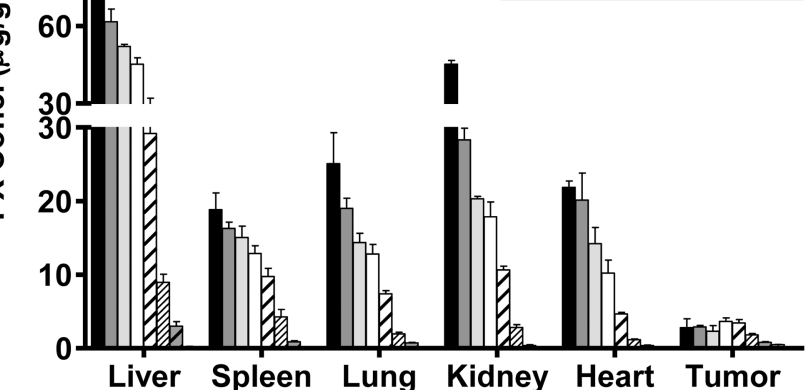

(D)

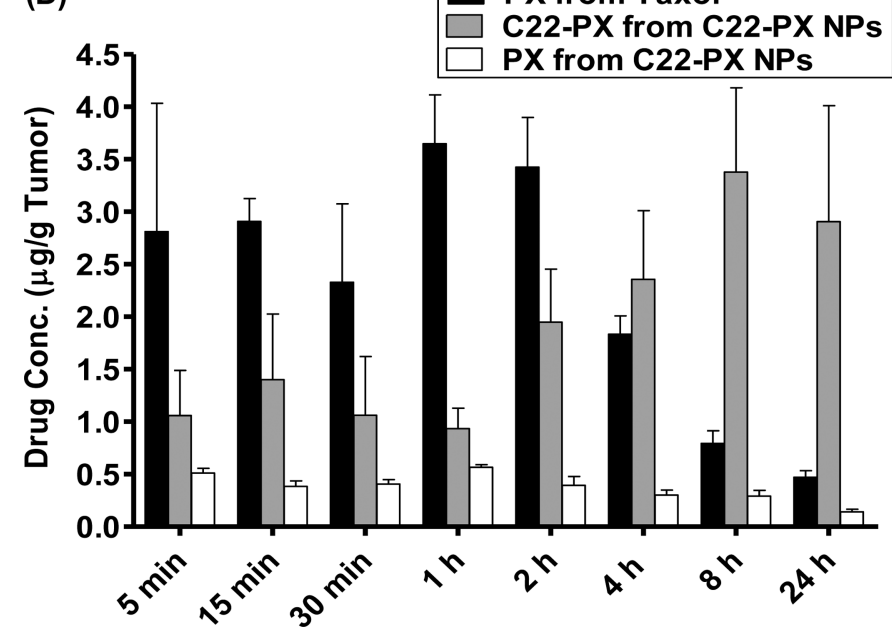

Figure 6.

Pharmacokinetic and biodistribution studies of Taxol and C22-PX NPs in a 4T1 mammary carcinoma mouse model at a dose of $10 \mathrm{mg}$ PX equivalent per $\mathrm{kg}$. BALB/c female mice $(\mathrm{n}=$ 3 per group) were s.c. injected with $1 \times 10^{6} 4 \mathrm{~T} 1$ cells and i.v. tail vein injected different formulations when the tumors reached to $200-300 \mathrm{~mm}^{3}$. (A) Drug concentrations in mouse plasma. (B) PX concentrations from Taxol. (C) C22-PX concentrations from C22-PX NPs. (D) Drug concentrations in tumor. Data are presented as the mean \pm SD. 
(A)
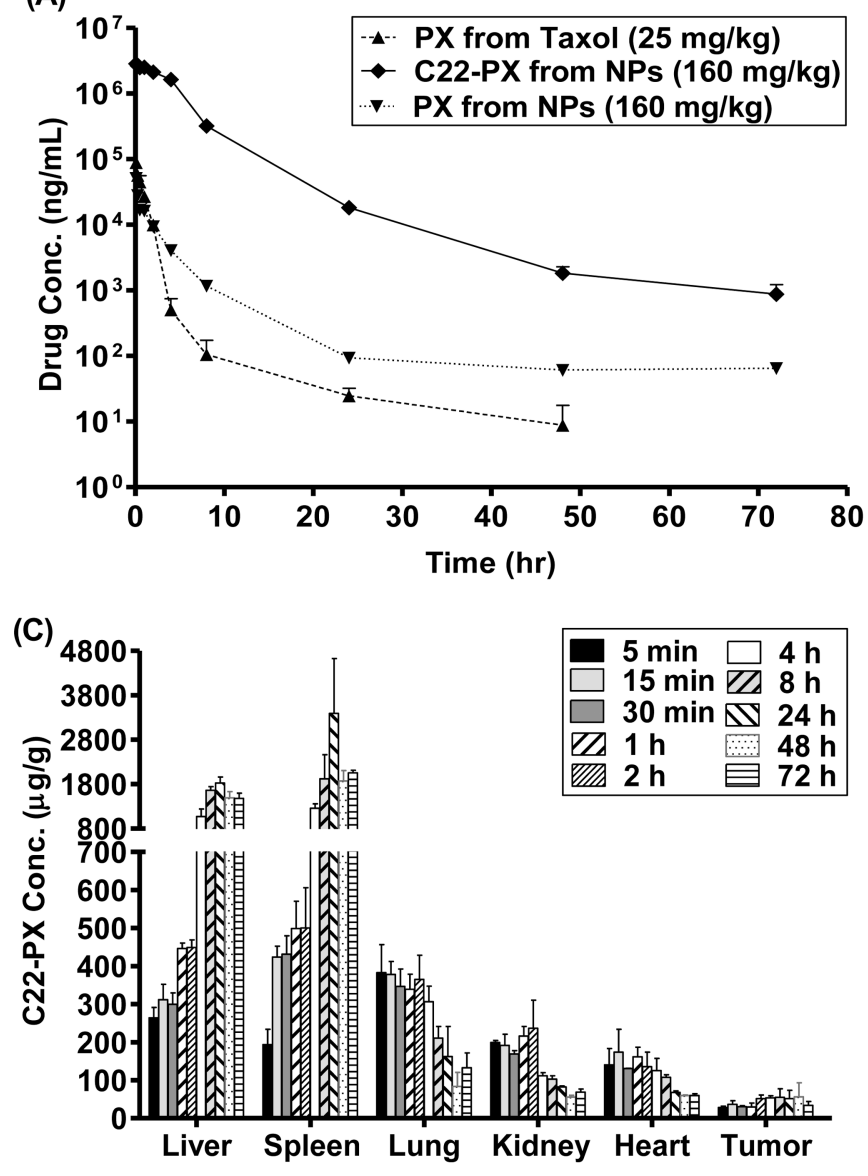

(B)
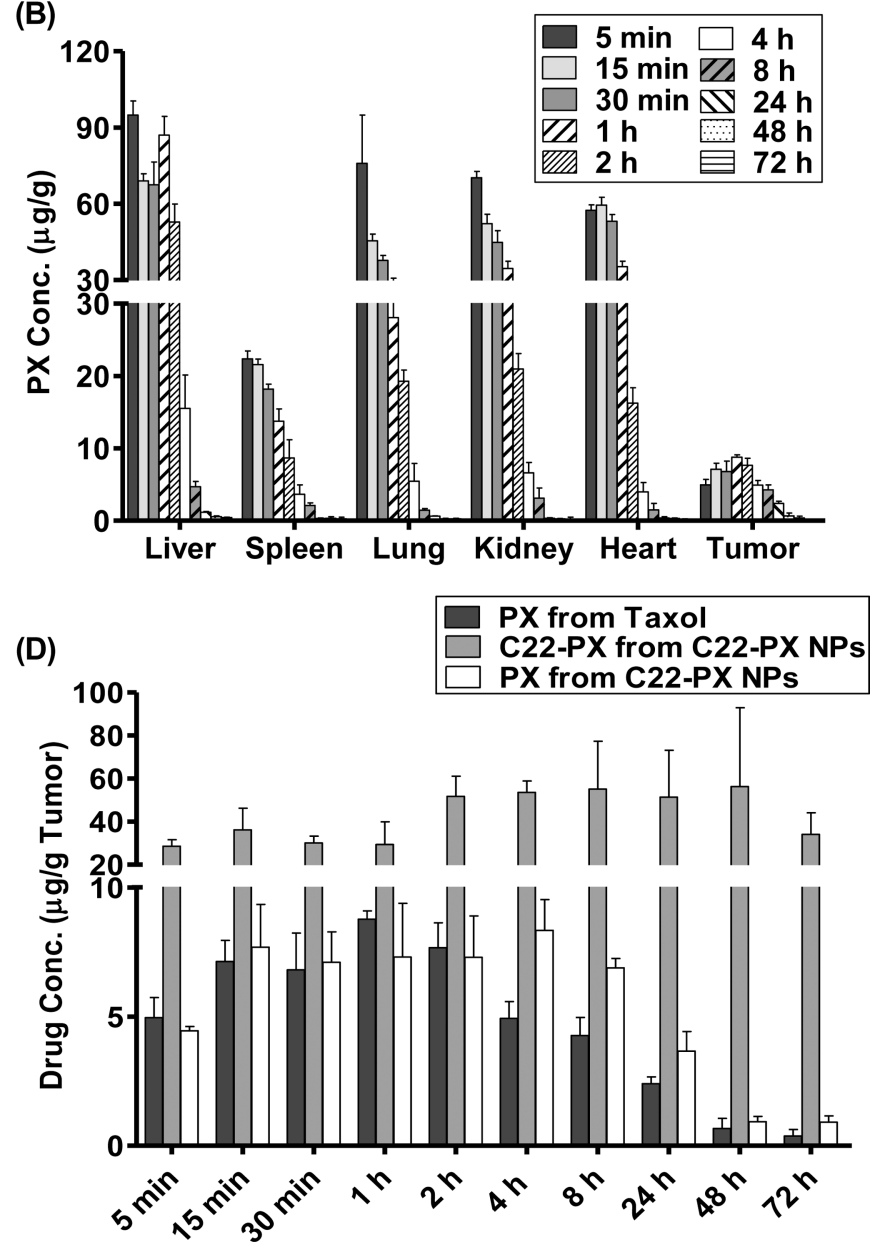

Figure 7.

Pharmacokinetic and biodistribution studies of Taxol and C22-PX NPs in a 4T1 mammary carcinoma mouse model at MTD. BALB/c female mice ( $\mathrm{n}=3$ per group) were s.c. injected with $1 \times 10^{6} 4 \mathrm{~T} 1$ cells and i.v. tail vein injected different formulations when the tumors reached to $200-300 \mathrm{~mm}^{3}$. (A) Drug concentrations in mouse plasma. (B) PX concentrations from Taxol. (C) C22-PX concentrations from C22-PX NPs. (D) Drug concentrations in tumor. Data are presented as the mean $\pm \mathrm{SD}$. 
(A)

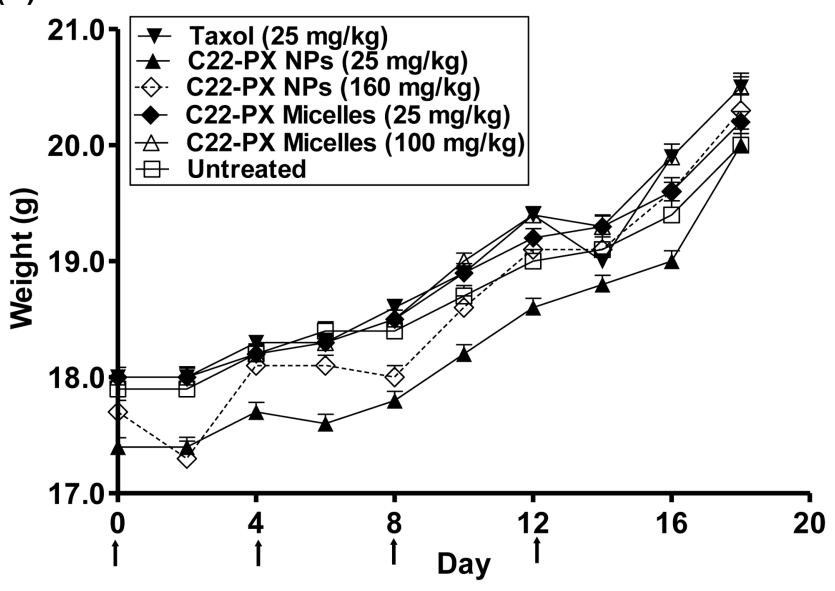

(B)

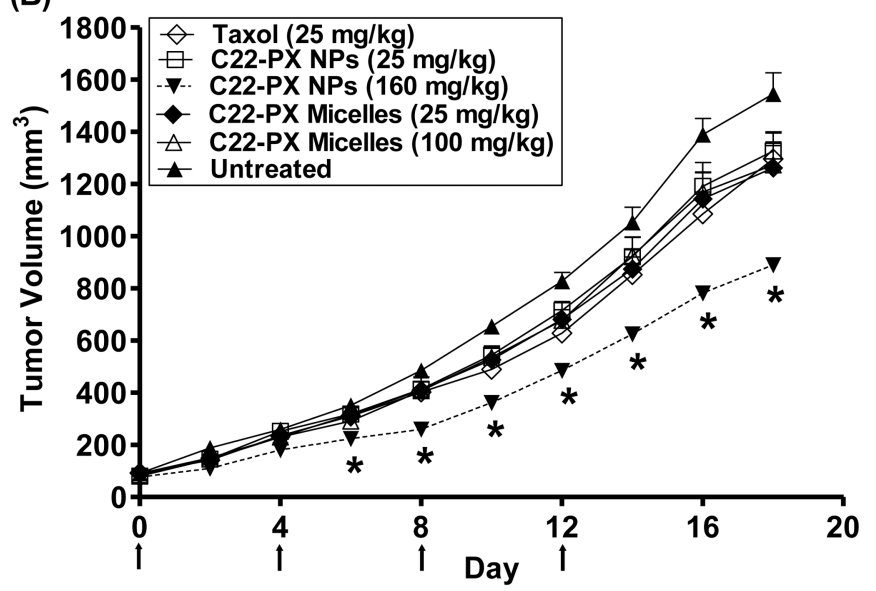

(C)

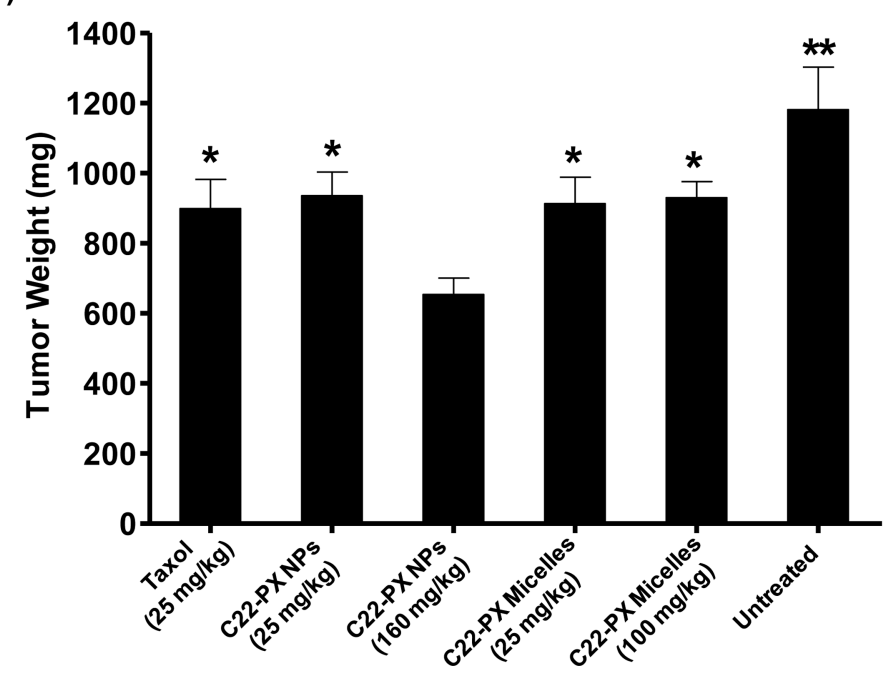

Figure 8.

In-vivo antitumor efficacy studies of Taxol, C22-PX NPs, and C22-PX micelles in a 4T1 mammary carcinoma mouse model. BALB/c female mice $(\mathrm{n}=9-10$ per group) were s.c. injected with $1 \times 10^{6} 4 \mathrm{~T} 1$ cells on Day (-5), and tail vein injected with different formulations starting from Day 0 with the treatment schedule of $\mathrm{Q} 4 \mathrm{~d} \times 4$. The mice weight and tumor sizes were measured every other day [tumor size was calculated as $1 / 2 \times{\text { (width })^{2}}^{2}$ $\times$ (length)]. All the mice were sacrificed on Day 18 and upon sacrifice the tumors of all the mice were removed and weighed. (A) Mice weight. (B) Tumor size. (C) Tumor weight. Data are presented as the mean \pm SEM. $\left(* \mathrm{p}<0.05,{ }^{* *} \mathrm{p}<0.01\right.$, compared to C22-PX NPs at the dose of $160 \mathrm{mg} / \mathrm{kg}$ ) 


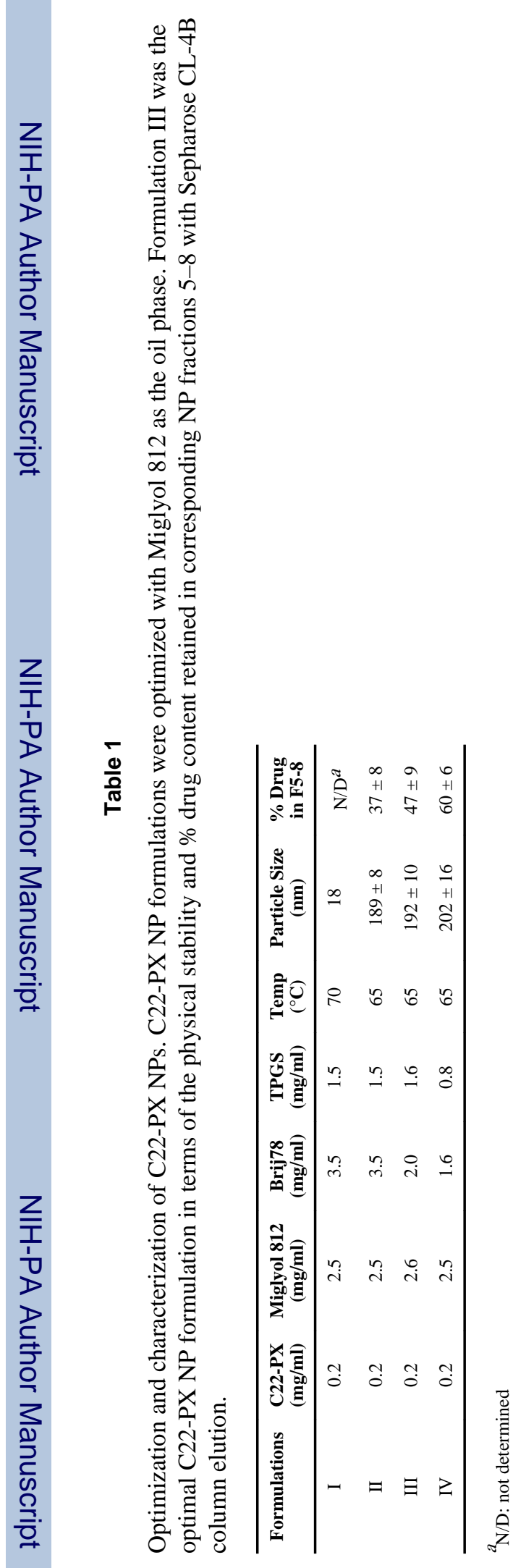

Cancer Lett. Author manuscript; available in PMC 2014 July 01. 


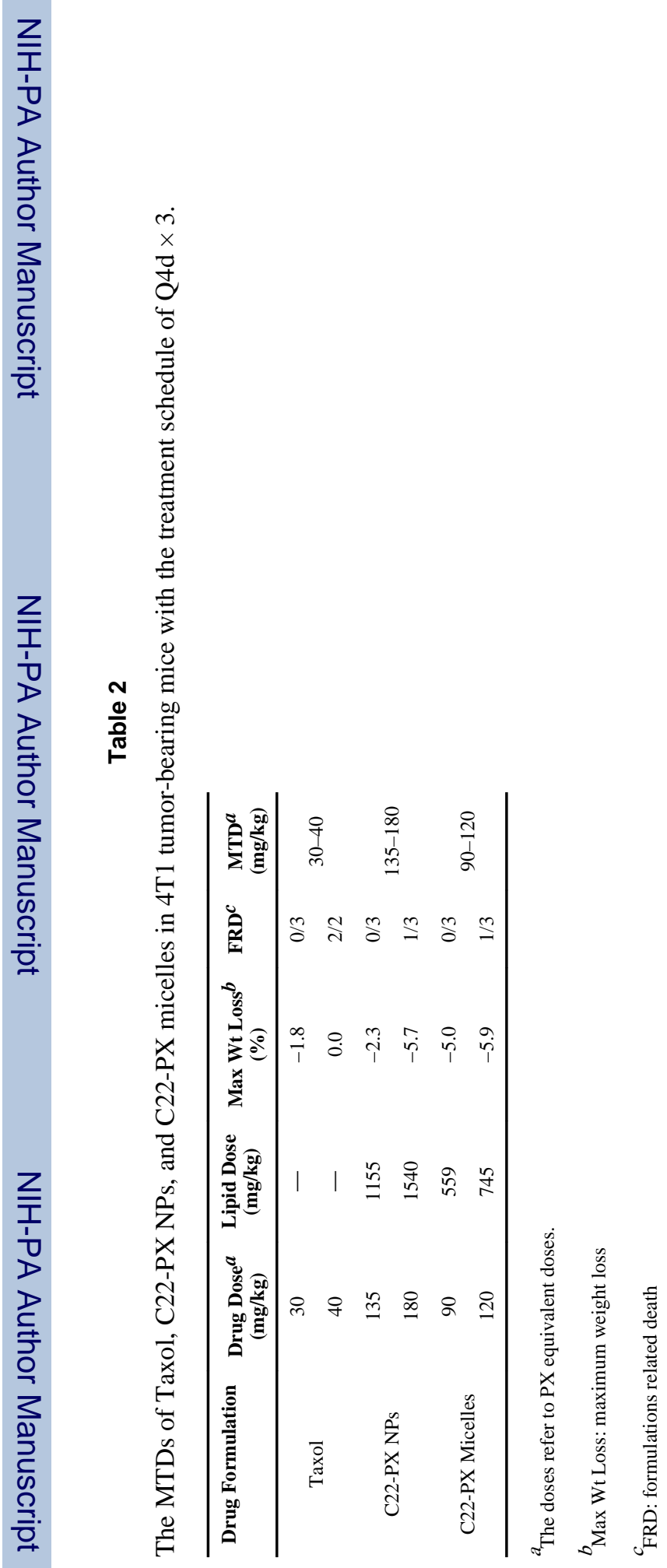




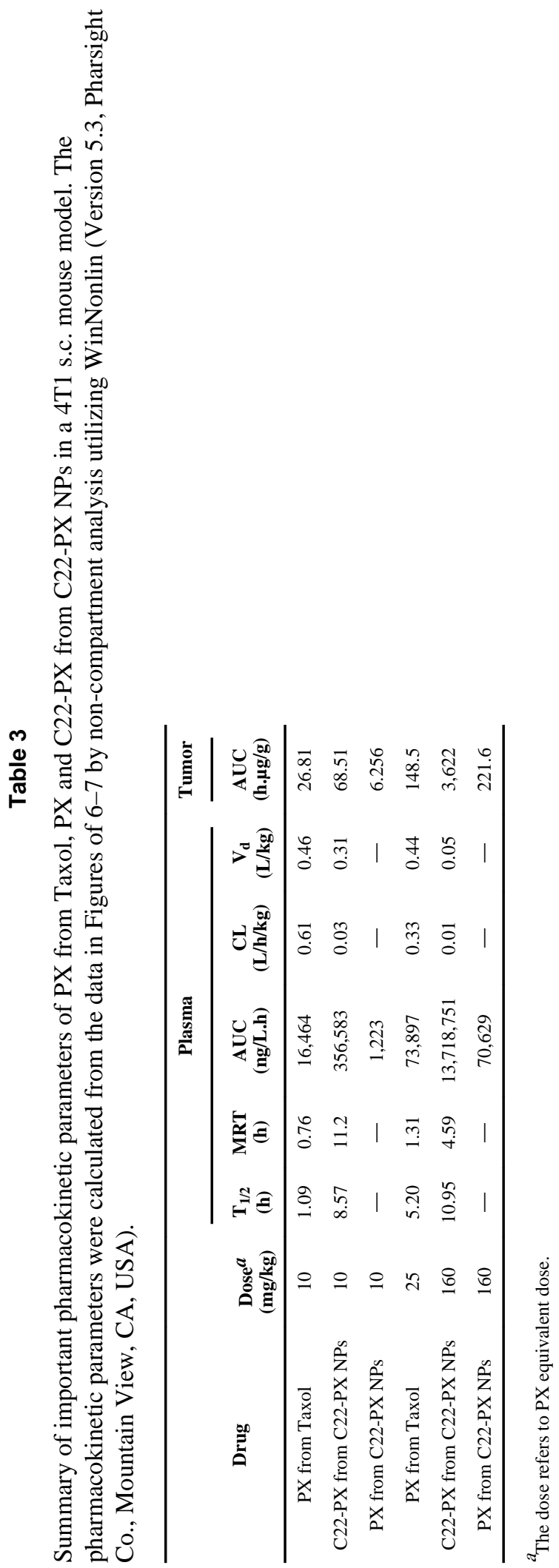

